\title{
Reassessment of the taxonomic relationships between closely related taxa of Papilionoideae
}

\author{
Maged Mahmoud Abou-El-Enain, Awatif Ibrahim El-Nahas, Abdemoniem Ibrahim \\ Aboel-Atta, Hanan *Helmy Latif, Shawkat Mahmoud Ahmed \& Nagwan Hamdy \\ Mohamed Atya \\ Biological and Geological Sciences Department, Faculty of Education, Ain Shams \\ University, Roxy, Heliopolis, P.C.11341, Cairo, Egypt \\ * Corresponding author: hananhelmy70@yahoo.com
}

\begin{abstract}
The present work was carried out to study some features of the family Papilonoideae. These features include seed protein profiles and morphology. These were used to reassess some taxonomic relationships between some closely related taxa of the Papilionoideae; identifying the similarities and dissimilarities between them based on the SDS-PAGE of seed protein characters; determination of the interrelationships and affinities between them based on current systems of classification by using numerical methods. Seed protein profiles produced by gel electrophoresis and the molecular mass in kilo-Dalton of their polypeptide subunits and the morphological features were recorded in 36 species belonging to 11 genera of the Papilionoideae (family: Fabaceae). Phenetic relationships of these species were established based on UPGMA-clustering applied to the produced 97 seed protein characters in combination with 173 morphological characters by using different modules of the NTSYS-pc 2.2 program package. The produced data were compared by measuring each of the tree distortion; from its relevant data matrix; and association to each other based on their cophenetic (ultrametric) correlation coefficients. The present data were useful in revealing new distinguishing characters, identifying the similarities/ differences and confirming the taxonomic interrelationships between the studied taxa based on current systems of classification.
\end{abstract}

Key words: SDS-PAGE, NTSYS, UPGMA, seed proteins, taxonomy, morphology.

\section{Introduction}

Papilionoideae (syn. Faboideae) is the largest, most diverse and widely distributed subfamily of Fabaceae sensu lato [1]. It comprises ca. 478 genera and 13,860 species and includes most of the familiar domesticated food plants, forage crops, genomic/genetic species and nitrogen fixation plants $[2$, $3]$. It is well represented in semi-arid to arid habitats of the world, with center of diversity in the temperate and tropical regions of South America. However, it is noticeably poorly represent or completely absent in the cool temperate habitats as in alpine regions and the understory of cool temperate forests [2].

Seeds of the subfamily contain about $50 \%$ protein from which the storage fraction represents nearly $50 \%$ of the total amount found in the seeds [4]. Storage protein is not sensitive to environmental fluctuations, being the third-hand copy of DNA and reflects the genetic make-up of the plant [5]. Seed protein banding patterns; produced by the polyacrylamide gel electrophoresis (PAGE) technique; constitute powerful attributes for plant taxonomy at various ranks specially 
when combined with morphology [6]. This fact prompted a considerable number of studies, many of which explained the inter/ intraspecific variations in individual genera e.g. Sesbania and Lathyrus [7,8], Onobrychis [9], Trifolium [10], Vicia [11] and Lathyrus [12,13].

The subfamily-wide studies in general have relatively been rare $[14,15]$, but there have been an increasing number of studies that have focused at tribal level, or on a number of tribes $[16,17,18,19,20,21,22]$. However, none of them had used the SDS-PAGE of seed protein criteria in relation to morphology. In the present study, such criteria are used with the aim of: refining the taxonomic relationships between some closely related taxa of the Papilionoideae; identifying the similarities and dissimilarities between them based on the SDS-PAGE of seed protein characters; determination of the interrelationships and affinities between them based on current systems of classification by using numerical methods.

\section{Material and Methods}

Seeds of 36 species belonging to 11 genera of the Papilionoideae were either collected from the Egyptian phytogeographic regions or provided by the Egyptian and German botanical gardens. The examined species, their source and taxonomic delimitation are given in Table 1. For the morphological examination, seeds were grown in a protected open area in the Botanical Garden of the Faculty of Education, Ain Shams University. Taxonomic

identification and nomenclature of Egyptian species follow Täeckholm (1974) [23] and Boulos (1999) [24], whereas those of the cultivated species follows Ball (1968) [25] and Bailey \& Bailey (1976) [26]. Identification was further confirmed by matching against dried specimens in the Herbaria of the
Faculty of Science, Ain Shams University (CAIA) and that of the Faculty of Education, Ain Shams University. Morphological description of the species was made from living flowering plants and vouchers are kept at the Herbarium of Biological and Geological Sciences Department, Faculty of Education, Ain Shams University.

For the electrophoretic analysis, seed protein was extracted by mixing $0.5 \mathrm{~g}$ of mature seeds with an equal weight of clean, sterile fine sand and powdered using a mortar and pestle and homogenized with $1 \mathrm{M}$ Tris- $\mathrm{HCl}$ buffer, $\mathrm{pH} 8.8$ in clean Eppendorf tube and left in refrigerator overnight. The extract was centrifuged at $3.000 \mathrm{rpm}$ for $10 \mathrm{~min}$. The supernatant (protein extract) was transferred to new tubes and immediately used for electrophoresis or kept in deep-freeze until use. For electrophoresis, $10 \mu \mathrm{l}$ of the extract were mixed with $5 \mu$ of a treatment buffer. Electrophoresis was carried out by the modified discontinuous sodium dodecyl sulfate PAGE (DISC SDS-PAGE) method (Laemmli, 1970) [27] using 12\% (w/v) acrylamide separating gel $(0.375 \mathrm{M}$ TRIS-HC1 buffer, $\mathrm{pH} 8.8)$ and $4 \%$ $(\mathrm{w} / \mathrm{v})$ acrylamide stacking gel $(0.125$ M TRIS-HC1, pH 6.8). The electrode buffer was TRIS-glycine (2.25 g TRIS and $10.8 \mathrm{~g}$ glycine per $750 \mathrm{ml}$ buffer solution, $\mathrm{pH} 8.3)$ with $0.1 \%(\mathrm{w} / \mathrm{v})$ SDS. Gels were then stained overnight in $0.1 \%(\mathrm{w} / \mathrm{v})$ Coomassie Brilliant Blue-R250 solution containing $40 \%$ $(\mathrm{v} / \mathrm{v})$ methanol and $10 \% \quad(\mathrm{v} / \mathrm{v})$ trichloracetic acid; destained in the same solution but without adding the dye. Gels were photographed, scanned and analyzed using Gel Doc 2000 BioRad system (Bio-Rad Laboratories, Hercules Co. USA). Total bands in the produced electrophorogram were scored and their biochemical masses $(\mathrm{Mr})$ in kilo-Dalton $(\mathrm{kDa})$ were 
calculated using standard protein marker.

For the numerical analyses, the data editor program NTedit 2.2; that is included in the program pakage of NTSYS-pc 2.2 (Rohlf, 2005) [28]; was used to prepare two sets of data matrices (proteins and morphology). Each of the studied species was considered as operational taxonomic unit (OTU) and numbered as indicated in Table (1). Multistate characters were transformed to two-state characters in coding (Sneath \& Sokal, 1973) [29]. A cluster analysis for the seed protein data set in combination with that of morphological data was performed by using the program NTSYS-pc 2.2 (Rohlf, 2005) [28]; as follows: The raw data matrix was standardized with STAND module; dissimilarity matrix was generated by SIMINT module based on Jaccard similarity distance (J). Clustering was performed using unweighted pair group method, arithmetic average (UPGMA) and represented in phenogram. In order to test reliability of results, the correlation coefficient $(r)$ value that measures the distortion between the produced phenogram and the relevant similarity matrix (Rohlf \& Sokal, 1981) [30] was estimated by using the program NTSYS-pc 2.2 (Rohlf, 2005) [28] as follows: The cophenetic (ultramatric) value matrix of the phenogram was computed using COPH module and compared to the related distance matrix using MXCOMP module.

\section{Result and Discussion}

The photograph of the produced SDSPAGE of seed protein profiles of the studied species is shown in Figure (1). The selected 270 characters (97 of protein and 173 of morphology); their codes and data matrix used in the numerical analysis are given in Appendix 1. UPGMA phenogram illustrating the relationships between the studied species based on each of the seed proteins, morphological and combined data sets are given in Figure (2). This phenogram revealed that; at reference line of 0.2 ; three major clusters (A, B \& C) are separated. The first cluster (A) comprises three minor groups $\left(a_{1}, a_{2} \& a_{3}\right)$ that are grouped together at the similarity level of 0.21 . The first minor group $\left(\mathrm{a}_{1}\right)$ comprises Anthyllis barba-jovis, Dorycnium hirsutum 1\&2, Genista linifolia and Colutea arborescens are distinguished at the level of 0.26 . The second group $\left(\mathrm{a}_{2}\right)$ comprises Hippocrepis multisiliquosa, Lotus corniculatus $1 \& 2, \quad$ L. pedunculatus, Lathyrus pratensis and $L$. aphaca that are grouped together at the similarity level of 0.28 and clustered with the group $\mathrm{a}_{1}$ at the 0.24 level. The third group $\left(a_{3}\right)$ comprises Vicia angustifolia, Vicia faba 1, $2 \& 3$ that are grouped together at the similarity level of 0.56 and clustered with the groups $a_{1} \& a_{2}$ at the 0.22 level.

The second major group (B) is represented by a single phenetic line that includes only Astragalus hamosus and is distinguished at the level of 0.20 . The third major cluster (C) comprises three minor groups $\left(\mathrm{c}_{1}, \mathrm{c}_{2}\right.$ \& $\left.\mathrm{c}_{3}\right)$. The first minor group $\left(\mathrm{c}_{1}\right)$ comprises Medicago citrina, $M$. lupulina, M. vardanis, M. orbicularis and $M$. sativa and is distinguished at the level of 0.47 . The second minor group $\left(\mathrm{c}_{2}\right)$ comprises Melilotus albus, $M$. graecus, $M$. indicus, $M$. messanensis and is distinguished at the level of 0.51 . The third minor group $\left(\mathrm{c}_{3}\right)$ comprises Trifolium alexandrinum, $T$. arvense, $T$. campestre $1 \& 2, T$. lappaceum, T. scabrum, T. fragiferum, T. infamia-ponertii, $T$. pratense, $T$. rubens and $T$. pignantii and is distinguished at the level of 0.39 . The produced correlation coefficient $(r)$ value of the reliability test for the used data set is 0.95 that reveals no 
significant distortion between the produced phenogram and its related similarity matrix.

[31] reported that, apertures and ornamentation of the pollen of the genus Anthyllis were very similar to those of Lotus and it was difficult to distinguish all the species in the two genera by pollen morphology. [32,33] classified Anthyllis in subtribe Anthyllidineae of the tribe Loteae. Their data revealed a close relationship between such genus and the genera Genista and Dorycnium. [34] supported these data and reported the taxonomic instability of the genus Genista to the extent that, he divided it into two genera (namely Teline beside Genista s. str.). In the present study, the examined species of these genera, namely Anthyllis barba-jovis, Dorycnium hirsutum (tribe Loteae), Genista linifolia (tribe Genistae) and Colutea arborescens (tribe Galegeae) were clustered in group $a_{1}$ of the cluster A. This was morphologically due to the shrub habit, broadly triangular stipules, capitates raceme flowers and teeth shorter than calyx tube. They also were characterized by the presence of protein bands with $\mathrm{Mr}$ of 106.41 and $32.45 \mathrm{kDa}$ (Character no. 178 and 225, Appendix 1), respectively; the absence of bands with Mr 117.71, 99.00 and 81.41 (Char. no. 174, 182 and 190), respectively. Thus, the present data partially support the conclusions of $[32,33]$. However, those of [34] needs a further study based on adequate number of samples. [35] reported the taxonomic similarities between Anthyllis with the related genera Dorycnium and Lotus for the first time; on the positive interaction with the associated root nodule bacteria which give a further support for the present conclusion.

[31] reported that, Hippocrepis was more similar to Lotus among taxa of the tribe Loteae in spite of their sharing two different types of pollen ornamentation. [36,37] pointed out that, the genera Lotus and Hippocrepis were restricted to the Old World with a highest number of species in the Mediterranean region. Of these, Lotus has non-lomentaceous fruits conforming to the traditional concept of Loteae while Hippocrepis was among the core of another tribe (Coronilleae). In the present study, Hippocrepis multisiliquosa, and Lotus corniculatus L. corniculatus2, $L$. pedunculatus were clustered in the group $a_{2}$ of the major cluster A. This was morphologically due to the herb habit, branched base stem and glabrous leaflets surface. They were characterized by the presence of protein bands with $\mathrm{Mr}$ of 106.41, 9.58 $\mathrm{kDa}$ (Char. no. 178 and 269), respectively; the absence of bands with Mr 25.89, 30.74 and 34.52 (Char. no. 237, 229 and

223), respectively. These data support the close relationship between both taxa and disagree with the conclusion of $[36,37]$.

[38,39] indicated the close relationships between Astragalus, Trifolium and Vicia based on phylogenetic analysis. In the present study, species of the three genera were clustered in different groups' $\mathrm{a}_{3}, \mathrm{~B}$ and $\mathrm{c}_{3}$, respectively. Astragalus hamosus was distinguished by linear bracts, tubular calyx shape and slightly curved pod and the presence of protein bands with $\mathrm{Mr}$ of $69.00,85.76$ and $63.22 \mathrm{kDa}$ (Char. no. 196, 188 and 201), respectively; the absence of bands with $\mathrm{Mr}$ of 102.97, 66.54 and $36.55 \mathrm{kDa}$ (Char. no. 180, 199 and 220), respectively. Vicia angustifolia, Vicia faba G461, Vicia faba L. Line2 and Vicia faba L. Line4 were clustered morphologically due to the annual longevity, glabrous stem surface, appressed hairy calyx teeth and dentate stipules margin. They were 
characterized by the presence of protein bands with $\mathrm{Mr}$ of 12.60, 11.03 $24.67 \mathrm{kDa}$ (Char. no. 260, 265 and 239), respectively; the absence of bands with $\mathrm{Mr}$ 31.41, 14.32 and 10.50 (Char. no. 227, 257 and 267), respectively. Trifolium alexandrinum, $T$. arvense, $T$. campestre, $T$. campestre2, $T$. lappaceum, $T$. fragiferum, $T$. infamia-ponertii, $T$. pignantii, $T$. pretense, $T$. rubens and $T$. scabrum were clustered together due to the herb habit, branched base, pubescent leaflets upper surface and entire stipules margin. They were characterized by the presence of protein bands with $\mathrm{Mr}$ of 117.71, 45.69 and $21.50 \mathrm{kDa}$ (Char. no. 174, 211 and 244), respectively; the absence of bands with Mr 105.10, 98.65 and 90.16 (Char. no. 179, 183 and 186), respectively. These data don't support the relationships reported by $[38,39]$.

[40,41] classified Melilotus into two subgenera; one includes $M$. albus and the other

subgenus included two sections that comprise all the other investigated species. [42] explained the deviation of $M$. albus based on the taxonomic similarity between species of the genera Melilotus and Medicago that have always been questioned. [43] constructed the phylogenetic relationships of both genera based on morphological characters and pointed out that, all species were strongly related to each other except for $M$. albus which was evolved early and that, its taxonomic position towards both genera was unclear. In the present study, Melilotus and Medicago were grouped in the same major cluster $\mathrm{C}$. Melilotus albus, M. graecus, $M$. indicus and $M$. messanensis were clustered in the group $\mathrm{c}_{2}$ morphologically due to the annual longevity, lanceolate stipules, obovateoblong corolla and glabrescent leaflets lower surface. They were characterized by the presence of protein bands with $\mathrm{Mr}$ of $81.41,36.55$ and $14.32 \mathrm{kDa}$ (Char. no. 190, 220 and 257), respectively; the absence of bands numbers with $\mathrm{Mr}$ 108.00, 100.91 and 53.81 (Char. no. 177, 181 and 204), respectively. Medicago citrina, $M$. lupulina, M. orbicularis, $M$. sativa and $M$. vardanis were clustered due to the adnate to petiole stipules, entire stipules and obovate leaflets. They were characterized by the presence of protein bands with $\mathrm{Mr}$ of 115.85, 45.69 and $15.51 \mathrm{kDa}$ (Char. no. 175, 211 and 254), respectively; the absence of bands numbers 176, 184 and 188 with $\mathrm{Mr}$ 111.00, 97.66 and 85.76, respectively. These data in one hand revealed the unity of Melilotus albus with the remaining studied species of its genus Melilotus. On the other hand, don't confirm the conclusion of [42] regarding the relationship of Melilotus and Medicago. 
Table 1. The examined Papilionoid taxa in the present study; their source and taxonomic delimitation.

\begin{tabular}{|c|c|c|c|}
\hline No. & Taxon & Source & Tribe \\
\hline 01 & Anthyllis barba-jovis $\mathbf{L}$. & BGBD & Loteae \\
\hline 02 & $\begin{array}{l}\text { Astragalus hamosus L. } \\
\text { (=Ankylobus hamosus } \text { (L.) Steven; Astragalus aegyptiacus Mill.; A. ancistron Pomel; A. arnoceras Bunge; A. brachyceras Ledeb.; A. buceras Willd.; A. } \\
\text { dorcoceras Bunge; A. embergeri Jahand. \& al.; A. oncocarpus Pomel; A. paui Pau; A. stribrnyi Velen.; A. taeckholmianus Oppenh.; A. volubilitanus Braun- } \\
\text { Blanq. \& Maire; Hamosa astragalus Medik.; Tragacantha brachyceras (Ledeb.) Kuntze; T. buceras (Willd.) Kuntze; T. hamosa (L.) Kuntze) }\end{array}$ & WILD $^{1}$ & Galegeae \\
\hline 03 & $\begin{array}{l}\begin{array}{l}\text { Colutea arborescens } \mathbf{L} . \\
(\equiv \text { Colutea arborea } \text { "sensu auct., p.p., Colutea brevialata Lange subsp. gallica forma brevialata). }\end{array} \\
\end{array}$ & BGBD & Galegeae \\
\hline 04 & $\begin{array}{l}\text { Dorycnium hirsutum }(\mathbf{L} .) \text { Ser.(1) } \\
\text { (=Bonjeanea hirsuta }(\text { L.) Rchb., Lotus hirsutus L., Lotus affinis Besser ex DC., Lotus candidus Mill., Bonjeanea cinerascens Jord. \& Fourr., Lotus } \\
\text { hemorroidalis Lam., Bonjeanea hirta Jord. \& Fourr., Bonjeanea italica Jord, Bonjeanea prostrata Jord. \& Fourr., Bonjeanea sericea (Sweet) Jord. \& } \\
\text { Fourr., Icon, Dorycnium sericeum Sweet, Dorycnium tomentosum G.Don, Bonjeanea venusta Jord. \& Fourr., Lotus intermedius Loisel., Lotus sericeus } \\
\text { DC.) }\end{array}$ & BGSA & Loteae \\
\hline 05 & D. hirsutum (L.) Ser. (2) & BGBD & Loteae \\
\hline 06 & $\begin{array}{l}\text { Genista linifolia L. } \\
\text { (=Teline gomerae (P.E.Gibbs \& Dingwall) Kunkel, Teline linifolia subsp. rosmarinifolia (Webb \& Berthel.) P.E.Gibbs \& D, Teline rosmarinifolia Webb \& } \\
\text { Berthel., Cytisus linifolius,Teline linifolia) }\end{array}$ & GBGA & Genistae \\
\hline 07 & $\begin{array}{l}\text { Hippocrepis multisiliquosa } \mathbf{L} . \\
(=\text { Hippocrepis ambigua (Rouy) Bellot, Hippocrepis confusa Pau, Hippocrepis multisiliquosa subsp. confusa (Pau) Maire) }\end{array}$ & GBGA & Loteae \\
\hline 08 & $\begin{array}{l}\text { Lathyrus aphaca L. } \\
\text { (= Orobus aphaca }(\text { L.) Doll., Aphaca vulgaris Presl., Lathyrus segetum Lam.) }\end{array}$ & WILD $^{2}$ & Vicieae \\
\hline 09 & $\begin{array}{l}\text { Lathyrus pratensis } \mathbf{L} . \\
(=\text { Orobus pratensis. })\end{array}$ & BGBD & Vicieae \\
\hline 10 & $\begin{array}{l}\text { Lotus corniculatus } \mathbf{L} \text {. } \\
\text { (=Lotus ambiguus Besser ex Spreng., Lotus ambiguus Spreng, Lotus arvensis } \text { Pers., Lotus balticus Miniaev, Lotus carpetanus Lacaita, Lotus caucasicus } \\
\text { Kuprian, Lotus caucasicus Kuprian., Lotus ciliatus sensu Schur, Lotus corniculatus L. var. crassifolia Fr., Lotus corniculatus L. var. kochii Chrtkova, Lotus } \\
\text { corniculatus } \text { L. var. maritimus Rupr., Lotus corniculatus subsp. major (Scop.) Gams, Lotus corniculatus var. arvensis (Pers.) Ser., Lotus corniculatus var. } \\
\text { glaber Opiz, Lotus corniculatus var. major (Scop.) Brand, Lotus corniculatus var.arvensis (Schkuhr) Ser. ex DC., Lotus filicaulis Durieu, Lotus frondosus } \\
\text { (Freyn) Kuprian, Lotus japonicus (Regel) K.larson, Lotus komarovii Miniaev, Lotus major Scop., Lotus olgae Klokov, Lotus peczoricus Miniaev and Ulle, } \\
\text { Lotus ruprechtii Miniaev, Lotus tauricus Juz., Lotus ucrainicus Klokov, Lotus zhegulensis Klokov) }\end{array}$ & $\begin{array}{ll}\text { BGBD } \\
\end{array}$ & Loteae \\
\hline 11 & $\begin{array}{l}\text { Lotus corniculatus } \mathbf{L} \text {. } \\
\text { (=Lotus ambiguus Besser ex Spreng., Lotus ambiguus Spreng, Lotus arvensis Pers., Lotus balticus Miniaev, Lotus carpetanus Lacaita, Lotus caucasicus } \\
\text { Kuprian, Lotus caucasicus Kuprian., Lotus ciliatus sensu Schur, Lotus corniculatus L. var. crassifolia Fr., Lotus corniculatus L. var. kochii Chrtkova, Lotus } \\
\text { corniculatus L. var. maritimus Rupr., Lotus corniculatus subsp. major (Scop.) Gams, Lotus corniculatus var. arvensis (Pers.) Ser., Lotus corniculatus var. } \\
\text { glaber Opiz, Lotus corniculatus var. major (Scop.) Brand, Lotus corniculatus var.arvensis (Schkuhr) Ser. ex DC., Lotus filicaulis Durieu, Lotus frondosus } \\
\text { (Freyn) Kuprian, Lotus japonicus (Regel) K.larson, Lotus komarovii Miniaev, Lotus major Scop., Lotus olgae Klokov, Lotus peczoricus Miniaev and Ulle, } \\
\text { Lotus ruprechtii Miniaev, Lotus tauricus Juz., Lotus ucrainicus Klokov, Lotus zhegulensis Klokov) }\end{array}$ & \begin{tabular}{|ll} 
BSST \\
\end{tabular} & Loteae \\
\hline
\end{tabular}




\begin{tabular}{|c|c|c|c|}
\hline 12 & $\begin{array}{l}\text { Lotus pedunculatus Cav. } \\
\text { (=Lotus granadensis Zertova, Lotus major Smith, Lotus trifoliolatus Eastw., Lotus uliginosus Schkuhr) }\end{array}$ & BGBD & Loteae \\
\hline 13 & $\begin{array}{l}\text { Medicago citrina (Font Quer) Greuter } \\
(=\text { Medicago arborea } \text { L. subspecies citrina Font Quer) }\end{array}$ & BGBD & Trifolieae \\
\hline 14 & $\begin{array}{l}\text { Medicago lupulina } \mathbf{L} . \\
(=\text { Medicago appenina Woods, Medicago wildenowii Merat, Medicago lupulina L. var. cupaniana (Guss.) Boiss, Medicago lupulina L. var. glandulosa } \\
\text { Neilr). }\end{array}$ & BGBD & Trifolieae \\
\hline 15 & $\begin{array}{l}\text { Medicago orbicularis }(\mathbf{L} .) \text { Bartal. } \\
\text { (= Medicago orbicularis }(\mathrm{L} .) \text { All., Medicago applanata Hornem, Medicago biancae (Urban) Pinto da Silva, Medicago cuneata J. Woods, Medicago } \\
\text { marginata Willd. }\end{array}$ & BGBD & Trifolieae \\
\hline 16 & $\begin{array}{l}\text { Medicago sativa L. } \\
\text { (=Medicago afganica (Bordere) Vassilcz; M. grandiflora (Grossh.)Vassilcz; M. ladak Vassilcz.; M. mesopotamica Vassilcz.; M. orientalis Vassilcz.; M. } \\
\text { polia (Brand) Vassilcz.; M. praesativa Sinskaya; M. sogdiana (Brand) Vassilcz; Trigonella upendrae H.J. Chowdhery \& R.R. Rao) }\end{array}$ & NRCD & Trifolieae \\
\hline 17 & $\begin{array}{l}\text { Medicago vardanis Vassilcz. } \\
\text { (= Medicago } \times \text { varia Martyn }=\text { Medicago sativa L. nothosubsp. varia }(\text { Martyn }) \text { Arcang). }\end{array}$ & BGBD & Trifolieae \\
\hline 18 & \begin{tabular}{|l|l|}
$\begin{array}{l}\text { Melilotus albus Medik. } \\
(=\text { Melilotus argutus Rchb., Melilotus albus var. annua Coe, Melilotus leucanthus W.D.J. Koch ex DC, Melilotus melanospermus Besser ex Ser., Melilotus } \\
\text { rugulosus } \text { Willd., Melilotus vulgaris (Hayne) Willd., Sertula alba (Medikus) Kuntze, Trifolium album (Medikus) Loisel., Trifolium vulgare Hayne. ) }\end{array}$ \\
\end{tabular} & BGBD & Trifolieae \\
\hline 19 & $\begin{array}{l}\begin{array}{l}\text { Melilotus graecus (Boiss. \& Spruner) Lassen } \\
(=\text { Trigonella graeca } \text { Boiss. \& Spruner) }\end{array} \\
\end{array}$ & BGBD & Trifolieae \\
\hline 20 & $\begin{array}{l}\text { Melilotus indicus (L.) All. } \\
\text { (=Melilotus bonplandii Ten.; M. indica (L.) All.; M. indicus (L.) All. subsp. permixtus (Jord.)Rouy; M. melilotus-indica Asch. \& Graebn.; M. melilotus- } \\
\text { indicus Asch. \& Graebn.; M. officinalis sensu Bojer; M. parviflora Desf.; M. parviflorus Desf.; M. permixtus Jord.; M. tommasinii Jord.; Trifolium indica } \\
\text { L.; T. indicum L.; T. indicus L.; T. melilotus-indica L.) }\end{array}$ & WILD $^{3}$ & Trifolieae \\
\hline 21 & $\begin{array}{l}\text { Melilotus messanensis (L.) All. } \\
\text { (=Melilotus sicula } \text { (Turra) B.D. Jacks.; M. siculus (Turra) B.D. Jacks.; Trifolium messanense L.) }\end{array}$ & WILD $^{4}$ & Trifolieae \\
\hline 22 & $\begin{array}{l}\text { Trifolium alexandrinum } \mathbf{L} . \\
(=\text { Trifolium alexandrium } \mathrm{L} .)\end{array}$ & NRCD & Trifolieae \\
\hline 23 & $\begin{array}{l}\text { Trifolium arvense } \mathbf{L} . \\
\text { (=Trifolium arenivagum Boreau, Trifolium brittingeri Opiz, Trifolium brachyodon (Celak.)A.Kern., Trifolium arvense subsp. gracile (Thuill.)Nyman, } \\
\text { Trifolium arvense var. gracile (Thuill.) DC., Trifolium eriocephalum Ledeb., Trifolium gracile Thuill., Trifolium longisetum Boiss. \& Balansa, Trifolium } \\
\text { capitulatum Pau, Trifolium agrestinum Boreau) }\end{array}$ & BGBD & Trifolieae \\
\hline 24 & $\begin{array}{l}\text { Trifolium campestre Schreb. } \\
\text { (=.Trifolium erythranthum (Griseb.)Halacsy, Trifolium pseudoprocumbens } \text { C.C.Gmel., Trifolium thionanthum Hausskn., Trifolium campestre var. lagrangei } \\
\text { (Boiss.)Zohary, Chrysaspis campestris (Schreb.)Desv., Trifolium agrarium L. p. p., Trifolium karatavicum Pavlov, Trifolium procumbens var. campestre } \\
\text { DC., Trifolium lagrangei Boiss., Trifolium pumilum Hossain, Trifolium procumbens L.) }\end{array}$ & $\begin{array}{l}\text { BGBD } \\
\end{array}$ & Trifolieae \\
\hline 25 & T. campestre2 & BGBD & Trifolieae \\
\hline 26 & $\begin{array}{l}\begin{array}{l}\text { Trifolium lappaceum } \mathbf{L} . \\
(=\text { Trifolium nervosum } \text { C. Presl, Trifolium rhodense Pampannini, Boll. Soc., Trifolium issajevii Khalilov ) }\end{array} \\
\end{array}$ & BGBD & Trifolieae \\
\hline 27 & 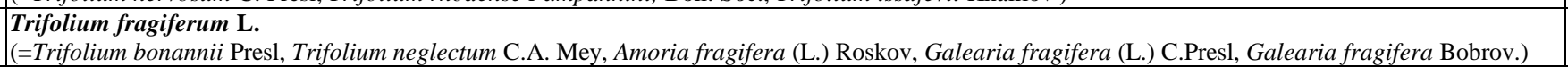 & BGBD & Trifolieae \\
\hline
\end{tabular}




\begin{tabular}{|c|c|c|c|}
\hline 28 & $\begin{array}{l}\text { Trifolium infamia-ponertii Greuter } \\
\text { (=Trifolium hybridum, Trifolium angustifolium subsp. gibellianum Pignatti, Trifolium angustifolium subsp. intermedium (Gibelli \& Belli) Arcang., } \\
\text { Trifolium angustifolium var. intermedium Gibelli \& Belli, Trifolium intermedium Guss.) }\end{array}$ & BGBD & Trifolieae \\
\hline 29 & Trifolium pignantii Fauché \& Chaub. & BGBD & Trifolieae \\
\hline 30 & \begin{tabular}{|l|l|} 
Trifolium pretense $\mathbf{L}$. subsp. Pretense \\
$(=$ Trifolium borysthenicum Gruner, Trifolium bracteatum Schousb., Trifolium lenkoranicum (Grossh.) Roskov, Trifolium pratense var. lenkoranicum \\
Grossh., Trifolium ukrainicum Opperman..)
\end{tabular} & BGBD & Trifolieae \\
\hline 31 & Trifolium rubens $\mathbf{L}$. & BGBD & Trifolieae \\
\hline 32 & Trifolium scabrum L. & BGBD & Trifolieae \\
\hline 33 & $\begin{array}{l}\text { Vicia angustifolia } \mathbf{L} . \\
\text { (=Vicia sativa subsp. nigra }(\text { L.) Ehrh.) }\end{array}$ & BGBD & Vicieae \\
\hline 34 & $\begin{array}{l}\text { Vicia faba G461 } \\
(=\text { Faba bona Medik.; F. equina Medik.; F. faba (L.) House; F. major Desf.; F. minor Roxb.; F. sativa Bernh.; F. vulgaris Moench; Orobus faba Brot.; } \\
\text { Vicia esculenta Salisb.; V. vulgaris Gray) }\end{array}$ & EMAG & Vicieae \\
\hline 35 & Vicia faba L. Line2 & EMAG & Vicieae \\
\hline 36 & Vicia faba L. Line4 & EMAG & Vicieae \\
\hline
\end{tabular}

BGBD= Botanic Garden of Berlin-Dahlem, Germany; BGSA= Botanic Garden of Salzburg, Germany; BSST= Berlin-Spandau station, Germany; EMAG= Egyptian Ministry

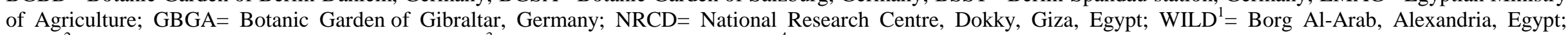
WILD $^{2}=$ Wadi Habes, Marsa-Matrouh, Egypt; WILD ${ }^{3}=$ Tabia, Rashied, Egypt; WILD ${ }^{4}=$ Boussaily, Rashied, Egypt. 


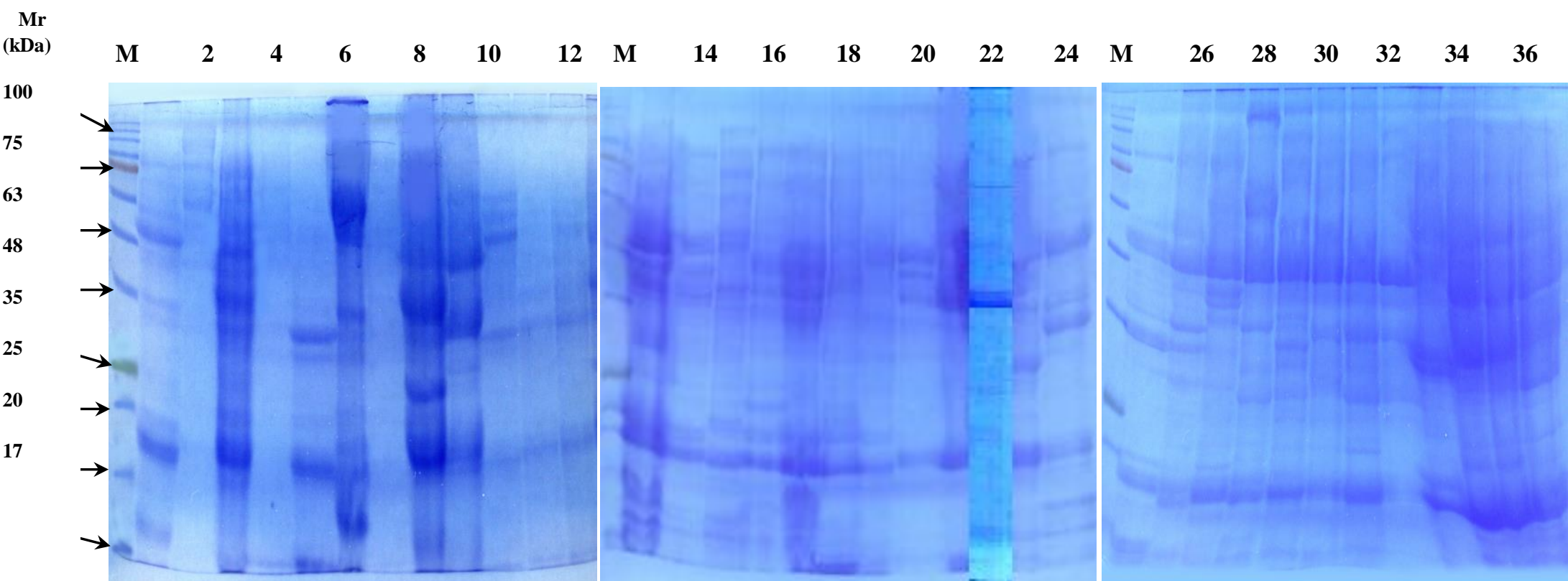

Fig. 1. The produced banding patterns of seed protein analysis using SDS-PAGE technique. (M. Marker, 1. Anthyllis barba-jovis, 2. Astragalus hamosus, 3. Colutea arborescens, 4. Dorycnium hirsutum(1), 5. Dorycnium hirsutum(2), 6. Genista linifolia, 7. Hippocrepis multisiliquosa, 8. Lathyrus aphaca, 9. Lathyrus pratensis, 10. Lotus corniculatus(1), 11. Lotus corniculatus(2), 12. Lotus pedunculatus, 13. Medicago citrina, 14. Medicago lupulina, 15. Medicago orbicularis, 16. Medicago sativa, 17. Medicago vardanis, 18. Melilotus albus, 19. Melilotus graecus, 20. Melilotus indicus, 21. Melilotus messanensis, 22. Trifolium alexandrinum, 23. Trifolium arvenses, 24. Trifolium campestre1, 25. Trifolium campestre2, 26. Trifolium lappaceum, 27. Trifolium fragiferum, 28. Trifolium infamia-ponertii, 29. Trifolium pignantii, 30. Trifolium pratenses, 31. Trifolium rubens, 32. Trifolium scabrum, 33. Vicia angustifolia, 34. Vicia fabal, 35. Vicia faba(2), 36. Vicia faba(3). 


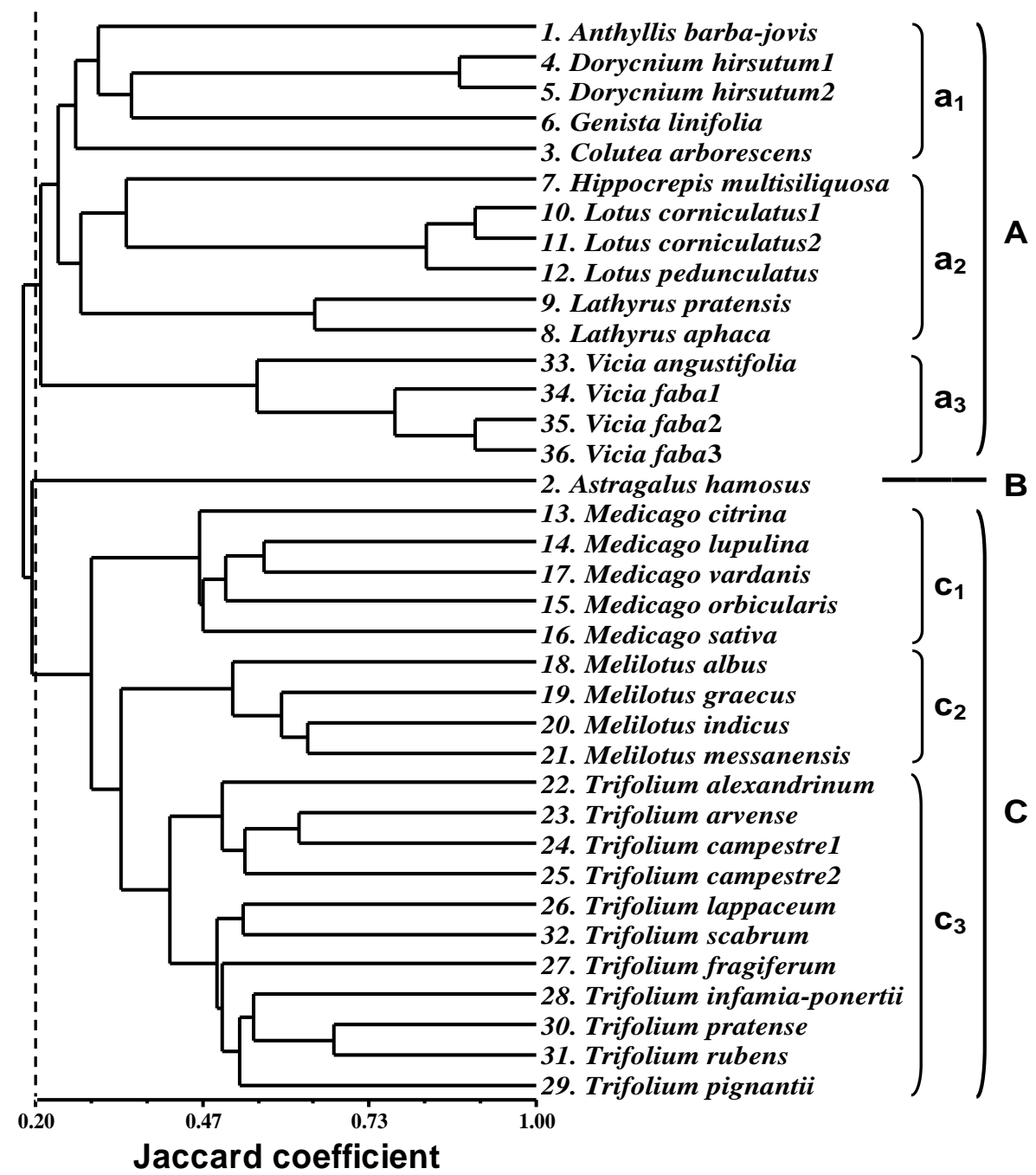

Fig. 2. The UPGMA phenogram showing clustering of the studied taxa based on combination of morphological and protein characters. Capital letters indicate the large groups, lowercase letters the ${ }_{4}$ subgroups. 
Appendix 1. Basic data matrix of the 270 studied characters used in the phenetic analysis of Papilionoid taxa. Numbers correspond to the species in Table 1.

\begin{tabular}{|c|c|c|c|c|c|c|c|c|c|c|c|c|c|c|c|c|c|c|c|c|c|c|c|c|c|c|c|c|c|c|c|c|c|c|c|c|c|}
\hline No. & Character IOUT's & $\begin{array}{l}\mathbf{0} \\
\mathbf{1}\end{array}$ & $\begin{array}{l}\mathbf{0} \\
\mathbf{2}\end{array}$ & $\begin{array}{l}\mathbf{0} \\
\mathbf{3}\end{array}$ & $\begin{array}{l}\mathbf{0} \\
4\end{array}$ & $\begin{array}{l}\mathbf{0} \\
\mathbf{5}\end{array}$ & $\begin{array}{l}\mathbf{0} \\
6\end{array}$ & $\begin{array}{l}\mathbf{0} \\
7\end{array}$ & $\begin{array}{l}\mathbf{0} \\
8\end{array}$ & $\begin{array}{l}\mathbf{0} \\
\mathbf{9}\end{array}$ & $\begin{array}{l}1 \\
\mathbf{0}\end{array}$ & $\begin{array}{l}1 \\
1\end{array}$ & $\begin{array}{l}1 \\
2\end{array}$ & $\begin{array}{l}1 \\
3\end{array}$ & $\begin{array}{l}1 \\
4\end{array}$ & $\begin{array}{l}1 \\
5\end{array}$ & $\begin{array}{l}1 \\
6\end{array}$ & $\begin{array}{l}1 \\
7\end{array}$ & $\begin{array}{l}1 \\
8\end{array}$ & $\begin{array}{l}1 \\
9\end{array}$ & $\begin{array}{l}2 \\
0\end{array}$ & $\begin{array}{l}2 \\
1\end{array}$ & $\begin{array}{l}2 \\
2\end{array}$ & $\begin{array}{l}2 \\
3\end{array}$ & $\begin{array}{l}2 \\
4\end{array}$ & $\begin{array}{l}2 \\
5\end{array}$ & $\begin{array}{l}2 \\
6\end{array}$ & $\begin{array}{l}2 \\
7\end{array}$ & $\begin{array}{l}2 \\
8\end{array}$ & $\begin{array}{l}2 \\
9\end{array}$ & $\begin{array}{l}3 \\
\mathbf{0}\end{array}$ & $\begin{array}{l}3 \\
1\end{array}$ & $\begin{array}{l}3 \\
2\end{array}$ & $\begin{array}{l}3 \\
3\end{array}$ & $\begin{array}{l}3 \\
4\end{array}$ & $\begin{array}{l}3 \\
5\end{array}$ & $\begin{array}{l}3 \\
6\end{array}$ \\
\hline & \multicolumn{37}{|c|}{ Morphological Characters } \\
\hline 1 & $\begin{array}{l}\text { Longevity: } \\
\text { Annual }\end{array}$ & 0 & 1 & 0 & 0 & 0 & 0 & 1 & 1 & 0 & 0 & 0 & 0 & 0 & 1 & 1 & 0 & 0 & 1 & 1 & 1 & 1 & 1 & 1 & 1 & 1 & 1 & 0 & 1 & 1 & 0 & 0 & 1 & 1 & 1 & 1 & 1 \\
\hline 2 & $\begin{array}{l}\text { Perennial } \\
\text { Habit: }\end{array}$ & 1 & 0 & 1 & 1 & 1 & 1 & 0 & 0 & 1 & 1 & 1 & 1 & 1 & 0 & 0 & 1 & 1 & 0 & 0 & 0 & 0 & 0 & 0 & 0 & 0 & 0 & 1 & 0 & 0 & 1 & 1 & 0 & 0 & 0 & 0 & 0 \\
\hline 3 & Herb & 0 & 1 & 0 & 0 & 0 & 0 & 1 & 1 & 1 & 1 & 1 & 1 & 0 & 1 & 1 & 1 & 1 & 1 & 1 & 1 & 1 & 1 & 1 & 1 & 1 & 1 & 1 & 1 & 1 & 1 & 1 & 1 & 1 & 1 & 1 & 1 \\
\hline 4 & $\begin{array}{l}\text { Shrub } \\
\text { Climbing: }\end{array}$ & 1 & 0 & 1 & 1 & 1 & 1 & 0 & 0 & 0 & 0 & 0 & 0 & 1 & 0 & 0 & 0 & 0 & 0 & 0 & 0 & 0 & 0 & 0 & 0 & 0 & 0 & 0 & 0 & 0 & 0 & 0 & 0 & 0 & 0 & 0 & 0 \\
\hline 5 & $\begin{array}{l}\text { Tendrils } \\
\text { Stem Shape: }\end{array}$ & 0 & 0 & 0 & 0 & 0 & 0 & 0 & 1 & 1 & 0 & 0 & 0 & 0 & 0 & 0 & 0 & 0 & 0 & 0 & 0 & 0 & 0 & 0 & 0 & 0 & 0 & 0 & 0 & 0 & 0 & 0 & 0 & 0 & 0 & 0 & 0 \\
\hline 6 & $\begin{array}{l}\text { crown arising from } \\
\text { leaf axils }\end{array}$ & 0 & 0 & 0 & 0 & 0 & 0 & 0 & 0 & 0 & 1 & 1 & 1 & 0 & 0 & 0 & 0 & 0 & 0 & 0 & 0 & 0 & 0 & 0 & 0 & 0 & 0 & 0 & 0 & 0 & 0 & 0 & 0 & 0 & 0 & 0 & 0 \\
\hline 7 & branched base & 1 & 1 & 0 & 0 & 0 & 1 & 1 & 1 & 1 & 1 & 1 & 1 & 1 & 0 & 0 & 0 & 0 & 1 & 1 & 1 & 1 & 1 & 1 & 1 & 1 & 1 & 1 & 1 & 1 & 1 & 1 & 1 & 1 & 1 & 1 & 1 \\
\hline 8 & Woody base & 0 & 0 & 1 & 1 & 1 & 0 & 0 & 0 & 0 & 0 & 0 & 0 & 0 & 1 & 1 & 1 & 1 & 0 & 0 & 0 & 0 & 0 & 0 & 0 & 0 & 0 & 0 & 0 & 0 & 0 & 0 & 0 & 0 & 0 & 0 & 0 \\
\hline 9 & $\begin{array}{l}\text { Slightly hirsute } \\
\text { Stem long: }\end{array}$ & 0 & 0 & 0 & 1 & 1 & 0 & 0 & 0 & 0 & 0 & 0 & 0 & 0 & 0 & 0 & 0 & 0 & 0 & 0 & 0 & 0 & 0 & 0 & 0 & 0 & 1 & 0 & 0 & 0 & 0 & 0 & 0 & 0 & 0 & 0 & 0 \\
\hline 10 & $\geq 10 \mathrm{~cm}$ & 0 & 0 & 0 & 1 & 1 & 0 & 0 & 0 & 0 & 0 & 0 & 0 & 0 & 0 & 0 & 0 & 0 & 0 & 0 & 0 & 0 & 0 & 0 & 0 & 0 & 0 & 0 & 0 & 0 & 0 & 0 & 0 & 0 & 0 & 0 & 0 \\
\hline 11 & $10-30 \mathrm{~cm}$ & 0 & 1 & 0 & 0 & 0 & 0 & 1 & 0 & 0 & 1 & 1 & 1 & 0 & 1 & 1 & 0 & 1 & 0 & 1 & 0 & 0 & ( & 1 & 1 & 1 & 1 & 1 & 1 & 1 & 1 & 0 & 1 & 0 & 0 & 0 & 0 \\
\hline 12 & $30-100 \mathrm{~cm}$ & 0 & 0 & 0 & 0 & 0 & 0 & 0 & 0 & 0 & 0 & 0 & 0 & 1 & 0 & 0 & 1 & 0 & 0 & 0 & 1 & 1 & 1 & 0 & 0 & 0 & 0 & 0 & 0 & 0 & 0 & 1 & 0 & 1 & 1 & 1 & 1 \\
\hline 13 & $100-400 \mathrm{~cm}$ & 1 & 0 & 1 & 0 & 0 & 1 & 0 & 1 & 1 & 0 & 0 & 0 & 0 & 0 & 0 & 0 & 0 & 0 & 0 & 0 & 0 & 0 & 0 & 0 & 0 & 0 & 0 & 0 & 0 & 0 & 0 & 0 & 0 & 0 & 0 & 0 \\
\hline 14 & $\begin{array}{l}<400 \mathrm{~cm} \\
\text { Stem Surface: }\end{array}$ & 0 & 0 & 0 & 0 & 0 & 0 & 0 & 0 & 0 & 0 & 0 & 0 & 0 & 0 & 0 & 0 & 0 & 1 & 0 & 0 & 0 & 0 & 0 & 0 & 0 & 0 & 0 & 0 & 0 & 0 & 0 & 0 & 0 & 0 & 0 & 0 \\
\hline 15 & Glabrous & 1 & 0 & 1 & 0 & 0 & 0 & 1 & 1 & 1 & 0 & 0 & 0 & 0 & 0 & 1 & 0 & 0 & 1 & 1 & 1 & 1 & 1 & 1 & 0 & 0 & 0 & 0 & 0 & 0 & 0 & 1 & 0 & 1 & 1 & 1 & 1 \\
\hline 16 & Pubescent & 0 & 1 & 0 & 1 & 1 & 0 & 0 & 0 & 0 & 0 & 0 & 0 & 1 & 1 & 0 & 0 & 1 & 0 & 0 & 0 & 0 & 0 & 0 & 0 & 0 & 0 & 0 & 0 & 0 & 0 & 0 & 0 & 0 & 0 & 0 & 0 \\
\hline 17 & $\begin{array}{l}\text { Hairy } \\
\text { Stipules Shape: }\end{array}$ & 0 & 0 & 0 & 0 & 0 & 1 & 0 & 0 & 0 & 1 & 1 & 1 & 0 & 0 & 0 & 1 & 0 & 0 & 0 & 0 & 0 & 0 & 0 & 1 & 1 & 1 & 1 & 1 & 1 & 1 & 0 & 1 & 0 & 0 & 0 & 0 \\
\hline 18 & Adnate to petiole & 0 & 0 & 0 & 0 & 0 & 0 & 0 & 0 & 0 & 1 & 1 & 1 & 1 & 1 & 1 & 1 & 1 & 0 & 0 & 0 & 0 & 0 & 0 & 0 & 0 & 0 & 0 & 0 & 1 & 0 & 0 & 0 & 0 & 0 & 0 & 0 \\
\hline 19 & Broadly triangular & 1 & 1 & 0 & 1 & 1 & 1 & 1 & 1 & 0 & 0 & 0 & 0 & 0 & 0 & 0 & 0 & 0 & 0 & 0 & 0 & 0 & 0 & 0 & 0 & 0 & 0 & 0 & 0 & 0 & 0 & 0 & 0 & 1 & 1 & 1 & 1 \\
\hline 20 & Green-veined & 0 & 0 & 0 & 0 & 0 & 0 & 0 & 0 & 0 & 0 & 0 & 0 & 0 & 0 & 0 & 0 & 0 & 0 & 0 & 0 & 0 & 1 & 0 & 0 & 0 & 1 & 0 & 0 & 0 & 0 & 0 & 1 & 0 & 0 & 0 & 0 \\
\hline 21 & Lanceolate & 0 & 0 & 0 & 0 & 0 & 0 & 0 & 0 & 1 & 0 & 0 & 0 & 1 & 1 & 0 & 0 & 1 & 1 & 1 & 1 & 1 & 0 & 0 & 0 & 0 & 0 & 1 & 1 & 0 & 0 & 1 & 1 & 0 & 0 & 0 & 0 \\
\hline 22 & Leafy & 0 & 0 & 0 & 0 & 0 & 0 & 0 & 1 & 0 & 0 & 0 & 0 & 0 & 0 & 0 & 0 & 0 & 0 & 0 & 0 & 0 & 0 & 0 & 0 & 0 & 0 & 0 & 0 & 0 & 0 & 0 & 0 & 0 & 0 & 0 & 0 \\
\hline 23 & Oblong & 0 & 0 & 0 & 0 & 0 & 0 & 0 & 0 & 0 & 0 & 0 & 0 & 0 & 0 & 0 & 0 & 0 & 0 & 0 & 0 & 0 & 1 & 1 & 0 & 0 & 0 & 0 & 0 & 0 & 0 & 0 & 0 & 0 & 0 & 0 & 0 \\
\hline 24 & Ovate & 0 & 0 & 0 & 0 & 0 & 0 & 0 & 0 & 0 & 0 & 0 & 0 & 0 & 0 & 0 & 1 & 0 & 0 & 0 & 0 & 0 & 0 & 0 & 1 & 1 & 0 & 0 & 0 & 0 & 1 & 0 & 0 & 0 & 0 & 0 & 0 \\
\hline 25 & dark spots at the & 0 & 0 & 0 & 0 & 0 & 0 & 1 & 0 & 0 & 0 & 0 & 0 & 0 & 0 & 0 & 0 & 0 & 0 & 0 & 0 & 0 & 0 & 0 & 0 & 0 & 0 & 0 & 0 & 0 & 0 & 0 & 0 & 0 & 0 & 0 & 0 \\
\hline
\end{tabular}


base

26 Hastate

Large arrow-

27 shaped

28 Finely laciniate

Stipules margin:

29 Dentate

30 Entire

Stipules surface:

31 Glabrou

Pilose

Scarious

Petiole length:

$34 \geq 0.5-2 \mathrm{~cm}$

$<2-5 \mathrm{~cm}$

$<5-10 \mathrm{~cm}$

$<10-12 \mathrm{~cm}$

Leaves Shape:

38 Imparipinnate

Paripinnate

40 odd pinnate

\section{Leaflets Pairs}

Number:

$41 \geq 4$

$42<4-9$

$3<9-17$

\section{Leaflets Shape:}

44 Deltoid

45 Lanceolate

46 Oblong

47 Obovate

48 Ovate

49 Elliptic

$\begin{array}{lllllllllllllllllllllllllllllllllllllllllll}0 & 0 & 0 & 0 & 0 & 0 & 0 & 1 & 1 & 0 & 0 & 0 & 0 & 0 & 0 & 0 & 0 & 0 & 0 & 0 & 0 & 0 & 0 & 0 & 0 & 0 & 0 & 0 & 0 & 0 & 0 & 0 & 0 & 0 & 0 & 0\end{array}$ $\begin{array}{llllllllllllllllllllllllllllllllllllllllllllllll}0 & 0 & 0 & 0 & 0 & 0 & 0 & 1 & 1 & 0 & 0 & 0 & 0 & 0 & 0 & 0 & 0 & 0 & 0 & 0 & 0 & 0 & 0 & 0 & 0 & 0 & 0 & 0 & 0 & 0 & 0 & 0 & 0 & 0 & 0 & 0\end{array}$ $\begin{array}{lllllllllllllllllllllllllllllllllllll}0 & 0 & 0 & 0 & 0 & 0 & 0 & 0 & 0 & 0 & 0 & 0 & 0 & 0 & 1 & 1 & 0 & 0 & 0 & 0 & 0 & 0 & 0 & 0 & 0 & 0 & 0 & 0 & 0 & 0 & 0 & 0 & 0 & 0 & 0 & 0\end{array}$ $\begin{array}{llllllllllllllllllllllllllllllllllllllll}0 & 0 & 0 & 0 & 0 & 0 & 0 & 0 & 0 & 0 & 0 & 0 & 0 & 0 & 0 & 0 & 0 & 0 & 1 & 1 & 1 & 0 & 0 & 0 & 0 & 0 & 0 & 0 & 0 & 0 & 0 & 0 & 1 & 1 & 1 & 1\end{array}$

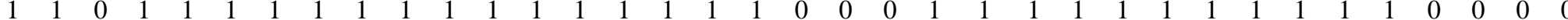
$\begin{array}{llllllllllllllllllllllllllllllllllllllllll}1 & 1 & 0 & 1 & 1 & 1 & 1 & 1 & 1 & 1 & 1 & 1 & 1 & 1 & 1 & 1 & 1 & 1 & 0 & 0 & 1 & 1 & 1 & 1 & 1 & 1 & 1 & 1 & 1 & 1 & 1 & 1 & 1 & 1 & 1 & 1\end{array}$ $\begin{array}{llllllllllllllllllllllllllllllllllllllllllllllll}0 & 0 & 0 & 0 & 0 & 0 & 0 & 0 & 0 & 0 & 0 & 0 & 0 & 0 & 0 & 0 & 0 & 0 & 1 & 1 & 0 & 0 & 0 & 0 & 0 & 0 & 0 & 0 & 0 & 0 & 0 & 0 & 0 & 0 & 0 & 0\end{array}$

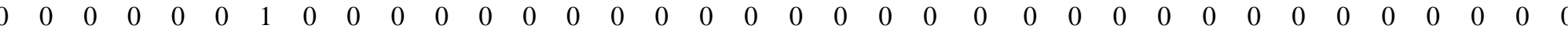

$\begin{array}{lllllllllllllllllllllllllllllllllllllllllllllll}0 & 0 & 0 & 1 & 1 & 1 & 1 & 0 & 0 & 1 & 1 & 1 & 0 & 0 & 1 & 1 & 1 & 1 & 0 & 0 & 0 & 0 & 0 & 1 & 1 & 1 & 0 & 1 & 1 & 0 & 1 & 1 & 1 & 1 & 1 & 1\end{array}$

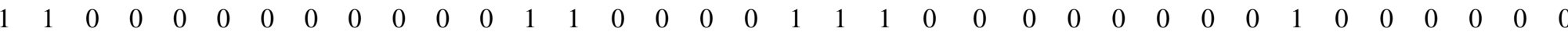
$\begin{array}{lllllllllllllllllllllllllllllllllllllllllll}0 & 0 & 0 & 0 & 0 & 0 & 0 & 0 & 0 & 0 & 0 & 0 & 0 & 0 & 0 & 0 & 0 & 0 & 0 & 0 & 0 & 1 & 0 & 0 & 0 & 0 & 1 & 0 & 0 & 0 & 0 & 0 & 0 & 0 & 0 & 0 \\ 0 & 0 & 1 & 0 & 0 & 0 & 0 & 0 & 0 & 0 & 0 & 0 & 0 & 0 & 0 & 0 & 0 & 0 & 0 & 0 & 0 & 0 & 0 & 0 & 0 & 0 & 0 & 0 & 0 & 0 & 0 & 0 & 0 & 0 & 0 & 0\end{array}$ $\begin{array}{llllllllllllllllllllllllllllllllllllllllllllllll}0 & 0 & 1 & 0 & 0 & 0 & 0 & 0 & 0 & 0 & 0 & 0 & 0 & 0 & 0 & 0 & 0 & 0 & 0 & 0 & 0 & 0 & 0 & 0 & 0 & 0 & 0 & 0 & 0 & 0 & 0 & 0 & 0 & 0 & 0 & 0\end{array}$ \begin{tabular}{llllllllllllllllllllllllllllllllllllllllll}
1 & 1 & 0 & 0 & 0 & 1 & 1 & 0 & 0 & 1 & 1 & 1 & 1 & 1 & 1 & 1 & 1 & 1 & 1 & 1 & 1 & 1 & 1 & 1 & 1 & 1 & 1 & 1 & 1 & 1 & 1 & 1 & 0 & 0 & 0 & 0 \\
\hline
\end{tabular}

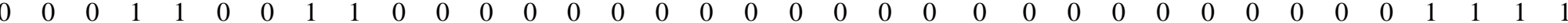

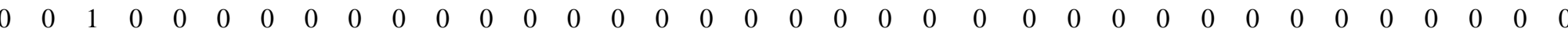

$\begin{array}{llllllllllllllllllllllllllllllllllllllllll}0 & 0 & 0 & 1 & 1 & 1 & 0 & 1 & 1 & 1 & 1 & 1 & 1 & 1 & 1 & 1 & 1 & 1 & 1 & 1 & 1 & 1 & 1 & 1 & 1 & 1 & 1 & 1 & 1 & 1 & 1 & 1 & 0 & 0 & 0 & 0 \\ 0 & 0 & 0 & 0 & 0 & 0 & 1 & 0 & 0 & 0 & 0 & 0 & 0 & 0 & 0 & 0 & 0 & 0 & 0 & 0 & 0 & 0 & 0 & 0 & 0 & 0 & 0 & 0 & 0 & 0 & 0 & 0 & 1 & 1 & 1 & 1\end{array}$

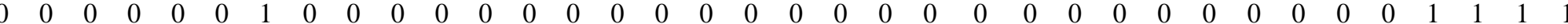
$\begin{array}{llllllllllllllllllllllllllllllllllllllllllll}1 & 1 & 1 & 0 & 0 & 0 & 0 & 0 & 0 & 0 & 0 & 0 & 0 & 0 & 0 & 0 & 0 & 0 & 0 & 0 & 0 & 0 & 0 & 0 & 0 & 0 & 0 & 0 & 0 & 0 & 0 & 0 & 0 & 0 & 0 & 0\end{array}$

$\begin{array}{lllllllllllllllllllllllllllllllllllllllllll}0 & 0 & 0 & 0 & 0 & 0 & 0 & 1 & 0 & 0 & 0 & 0 & 0 & 0 & 0 & 0 & 0 & 0 & 0 & 0 & 0 & 0 & 0 & 0 & 0 & 0 & 0 & 0 & 0 & 0 & 0 & 0 & 0 & 0 & 0 & 0\end{array}$ $\begin{array}{lllllllllllllllllllllllllllllllllllllllllllllllll}0 & 0 & 0 & 0 & 0 & 1 & 0 & 0 & 1 & 0 & 0 & 0 & 0 & 0 & 0 & 0 & 0 & 0 & 0 & 0 & 0 & 0 & 0 & 0 & 0 & 0 & 0 & 1 & 0 & 0 & 0 & 0 & 0 & 0 & 0 & 0\end{array}$

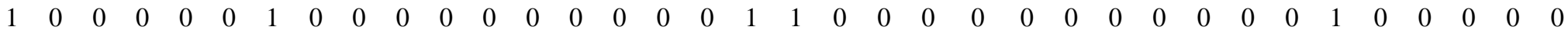

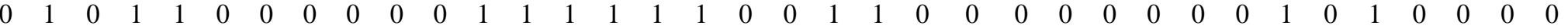
$\begin{array}{llllllllllllllllllllllllllllllllllllllllll}0 & 0 & 1 & 0 & 0 & 0 & 0 & 0 & 0 & 0 & 0 & 0 & 0 & 0 & 0 & 0 & 0 & 0 & 0 & 0 & 0 & 0 & 0 & 0 & 0 & 1 & 0 & 0 & 0 & 0 & 0 & 0 & 1 & 1 & 1 & 1\end{array}$ $\begin{array}{llllllllllllllllllllllllllllllllllllllllll}0 & 0 & 0 & 0 & 0 & 0 & 0 & 0 & 0 & 1 & 1 & 0 & 0 & 0 & 0 & 0 & 0 & 0 & 0 & 0 & 0 & 1 & 1 & 1 & 1 & 0 & 1 & 0 & 1 & 0 & 0 & 0 & 0 & 0 & 0 & 0\end{array}$ 
Leaflets Margin:

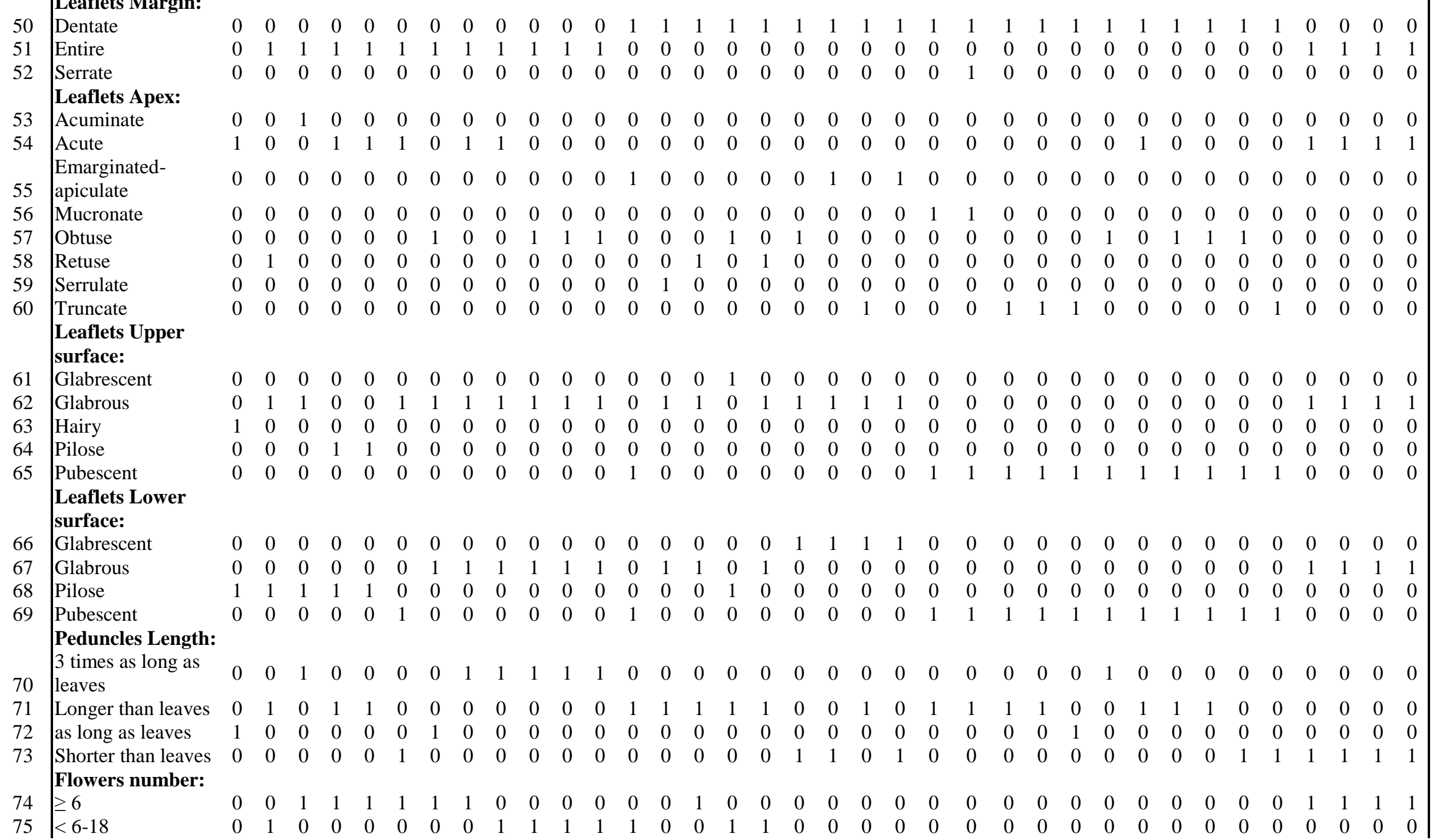




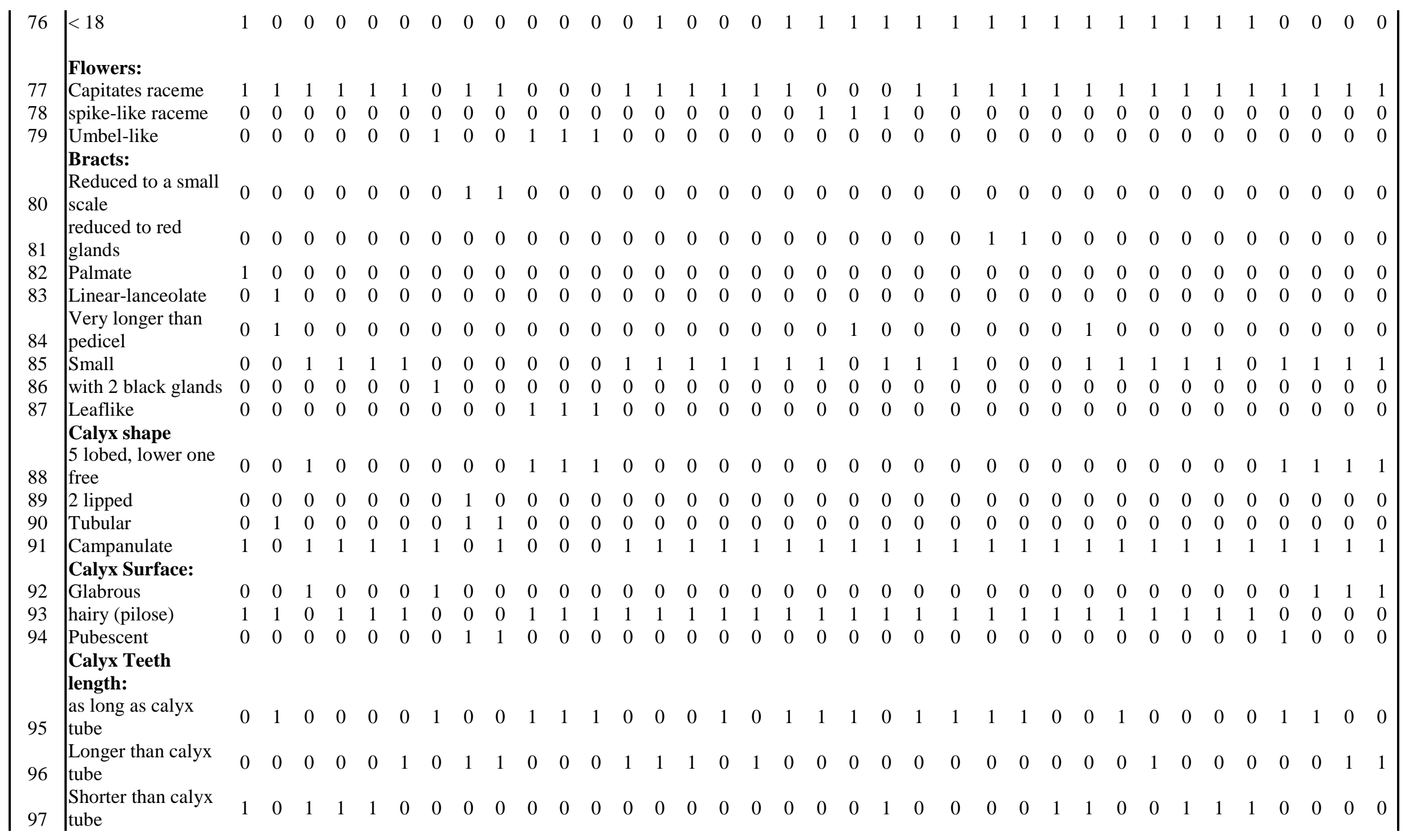


Calyx Teeth shape

\begin{tabular}{|c|c|c|c|c|c|c|c|c|c|c|c|c|c|c|c|c|c|c|c|c|c|c|c|c|c|c|c|c|c|c|c|c|c|c|c|c|c|}
\hline & Calyx Teeth shape & & & & & & & & & & & & & & & & & & & & & & & & & & & & & & & & & & & & \\
\hline 98 & Acuminate & 0 & 0 & 0 & 0 & 0 & 0 & 0 & 0 & 0 & 0 & 0 & 0 & 0 & 0 & 0 & 0 & 0 & 0 & 0 & 0 & 1 & 0 & 0 & 0 & 0 & 0 & 0 & 0 & 0 & 0 & 0 & 0 & 0 & 0 & 0 & 0 \\
\hline 99 & Acute & 0 & 0 & 0 & 0 & 0 & 0 & 1 & 0 & 0 & 1 & 1 & 1 & 0 & 0 & 0 & 0 & 0 & 1 & 0 & 0 & 0 & 0 & 0 & 0 & 0 & 0 & 0 & 0 & 0 & 0 & 0 & 0 & 1 & 1 & 1 & 1 \\
\hline 100 & Appressed hairy & 1 & 0 & 0 & 1 & 1 & 0 & 0 & 0 & 0 & 0 & 0 & 0 & 0 & 0 & 0 & 0 & 0 & 1 & 1 & 1 & 1 & 1 & 1 & 1 & 1 & 1 & 1 & 1 & 1 & 1 & 1 & 1 & 0 & 0 & 0 & 0 \\
\hline 101 & Lanceolate & 0 & 0 & 0 & 0 & 0 & 1 & 0 & 1 & 1 & 0 & 0 & 0 & 1 & 1 & 1 & 1 & 1 & 0 & 0 & 0 & 0 & 0 & 0 & 0 & 0 & 0 & 0 & 0 & 0 & 0 & 0 & 1 & 0 & 0 & 0 & 0 \\
\hline 102 & spinulose tips & 0 & 0 & 0 & 0 & 0 & 0 & 0 & 0 & 0 & 0 & 0 & 0 & 0 & 0 & 0 & 0 & 0 & 0 & 0 & 0 & 0 & 1 & 1 & 0 & 0 & 0 & 0 & 0 & 0 & 0 & 0 & 0 & 0 & 0 & 0 & 0 \\
\hline 103 & Subulate & 0 & 1 & 0 & 0 & 0 & 0 & 0 & 0 & 0 & 0 & 0 & 0 & 0 & 0 & 0 & 1 & 0 & 0 & 0 & 0 & 1 & 1 & 1 & 1 & 1 & 1 & 0 & 0 & 0 & 0 & 0 & 0 & 0 & 0 & 0 & 0 \\
\hline 104 & $\begin{array}{l}\text { Deltoid } \\
\text { Corolla Length: }\end{array}$ & 0 & 0 & 1 & 0 & 0 & 0 & 0 & 0 & 0 & 0 & 0 & 0 & 0 & 0 & 0 & 0 & 0 & 0 & 0 & 1 & 0 & 0 & 0 & 0 & 0 & 0 & 0 & 0 & 0 & 0 & 0 & 0 & 0 & 0 & 0 & 0 \\
\hline 105 & Longer than calyx & 1 & 1 & 0 & 1 & 1 & 0 & 1 & 1 & 1 & 0 & 0 & 0 & 0 & 0 & 0 & 0 & 0 & 0 & 0 & 0 & 0 & 0 & 0 & 0 & 0 & 0 & 1 & 0 & 1 & 1 & 1 & 0 & 0 & 0 & 0 & 0 \\
\hline 06 & $\begin{array}{l}\text { twice as long as } \\
\text { calyx }\end{array}$ & 0 & 0 & 0 & 0 & 0 & 0 & 0 & 0 & 0 & 0 & 0 & 0 & 0 & 0 & 1 & 0 & 0 & 1 & 1 & 1 & 1 & 1 & 1 & 1 & 1 & 0 & 0 & 0 & 0 & 0 & 0 & 0 & 0 & 0 & 0 & 0 \\
\hline 107 & $\begin{array}{l}>\text { twice as long as } \\
\text { calyx }\end{array}$ & 0 & 0 & 1 & 0 & 0 & 0 & 0 & 0 & 0 & 1 & 1 & 1 & 0 & 0 & 0 & 1 & 0 & 0 & 0 & 0 & 0 & 0 & 0 & 0 & 0 & 0 & 0 & 0 & 0 & 0 & 0 & 0 & 1 & 1 & 1 & 1 \\
\hline 08 & $\begin{array}{l}\text { Shorter than the } \\
\text { calyx }\end{array}$ & 0 & 0 & 0 & 0 & 0 & 0 & 0 & 0 & 0 & 0 & 0 & 0 & 0 & 0 & 0 & 0 & 0 & 0 & 0 & 0 & 0 & 0 & 0 & 0 & 0 & 1 & 0 & 1 & 0 & 0 & 0 & 0 & 0 & 0 & 0 & 0 \\
\hline 109 & $\begin{array}{l}\text { as long as calyx } \\
\text { Corolla color: }\end{array}$ & 0 & 0 & 0 & 0 & 0 & 1 & 0 & 0 & 0 & 0 & 0 & 0 & 1 & 1 & 0 & 0 & 1 & 0 & 0 & 0 & 0 & 0 & 0 & 0 & 0 & 0 & 0 & 0 & 0 & 0 & 0 & 1 & 0 & 0 & 0 & 0 \\
\hline 110 & White & 1 & 0 & 0 & 0 & 0 & 0 & 0 & 0 & 0 & 0 & 0 & 0 & 0 & 0 & 0 & 0 & 0 & 1 & 0 & 0 & 0 & 1 & 1 & 0 & 0 & 1 & 1 & 0 & 0 & 0 & 0 & 1 & 0 & 1 & 1 & 1 \\
\hline 111 & Yellowish-white & 0 & 1 & 0 & 0 & 0 & 0 & 0 & 0 & 0 & 0 & 0 & 0 & 0 & 0 & 0 & 0 & 0 & 0 & 0 & 0 & 0 & 0 & 0 & 0 & 0 & 0 & 0 & 0 & 1 & 0 & 0 & 0 & 0 & 0 & 0 & 0 \\
\hline 112 & Yellow & 0 & 0 & 1 & 0 & 0 & 0 & 1 & 1 & 1 & 1 & 1 & 0 & 1 & 1 & 1 & 0 & 0 & 0 & 1 & 1 & 1 & 0 & 0 & 1 & 1 & 0 & 0 & 0 & 0 & 0 & 0 & 0 & 0 & 0 & 0 & 0 \\
\hline 13 & $\begin{array}{l}\text { white veined } \\
\text { pinkish-red }\end{array}$ & 0 & 0 & 0 & 1 & 1 & 0 & 0 & 0 & 0 & 0 & 0 & 0 & 0 & 0 & 0 & 0 & 0 & 0 & 0 & 0 & 0 & 0 & 0 & 0 & 0 & 0 & 0 & 0 & 0 & 0 & 0 & 0 & 1 & 0 & 0 & 0 \\
\hline 114 & Golden & 0 & 0 & 0 & 0 & 0 & 1 & 0 & 0 & 0 & 0 & 0 & 1 & 0 & 0 & 0 & 0 & 0 & 0 & 0 & 0 & 0 & 0 & 0 & 0 & 0 & 0 & 0 & 0 & 0 & 0 & 0 & 0 & 0 & 0 & 0 & U \\
\hline 115 & $\begin{array}{l}\text { Purple } \\
\text { Corolla Shape: }\end{array}$ & 0 & 0 & 0 & 0 & 0 & 0 & 0 & 0 & 0 & 0 & 0 & 0 & 0 & 0 & 0 & 1 & 1 & 0 & 0 & 0 & 0 & 0 & 0 & 0 & 0 & 0 & 0 & 1 & 0 & 1 & 1 & 0 & 0 & 0 & 0 & 0 \\
\hline 116 & Obovate-oblong & 0 & 1 & 1 & 0 & 0 & 0 & 0 & 0 & 0 & 0 & 0 & 0 & 0 & 0 & 0 & 0 & 0 & 1 & 1 & 1 & 1 & 0 & 0 & 0 & 0 & 0 & 0 & 0 & 0 & 0 & 0 & 0 & 0 & 0 & 0 & 0 \\
\hline 117 & Ovate & 1 & 0 & 0 & 0 & 0 & 1 & 0 & 0 & 0 & 0 & 0 & 0 & 0 & 0 & 0 & 0 & 0 & 0 & 0 & 0 & 0 & 0 & 0 & 0 & 0 & 0 & 0 & 0 & 0 & 0 & 0 & 0 & 1 & 1 & 1 & 1 \\
\hline 118 & keel incurved & 1 & 0 & 0 & 1 & 1 & 0 & 1 & 1 & 1 & 1 & 1 & 1 & 0 & 0 & 0 & 0 & 0 & 0 & 0 & 0 & 0 & 0 & 0 & 0 & 0 & 0 & 0 & 0 & 0 & 0 & 0 & 0 & 0 & 0 & 0 & 0 \\
\hline & $\begin{array}{l}\text { Wing spur in keel } \\
\text { invagin. }\end{array}$ & 0 & 0 & 0 & 0 & 0 & 0 & 0 & 0 & 0 & 0 & 0 & 0 & 1 & 1 & 1 & 1 & 1 & 0 & 0 & 0 & 0 & 0 & 0 & 0 & 0 & 0 & 0 & 0 & 0 & 0 & 0 & 0 & 0 & 0 & 0 & 0 \\
\hline & $\begin{array}{l}\text { Wing adhering by } \\
\text { tooth }\end{array}$ & 0 & 0 & 0 & 0 & 0 & 0 & 0 & 0 & 0 & 0 & 0 & 0 & 0 & 0 & 0 & 0 & 0 & 1 & 1 & 1 & 1 & 0 & 0 & 0 & 0 & 0 & 0 & 0 & 0 & 0 & 0 & 0 & 0 & 0 & 0 & 0 \\
\hline & Wing free & 0 & 0 & 0 & 0 & 0 & 0 & 0 & 1 & 1 & 1 & 1 & 1 & 0 & 0 & 0 & 0 & 0 & 0 & 0 & 0 & 0 & 0 & 0 & 0 & 0 & 0 & 0 & 0 & 0 & 0 & 0 & 0 & 1 & 1 & 1 & 1 \\
\hline & Wing oblong-ovate & 0 & 0 & 0 & 0 & 0 & 0 & 0 & 0 & 0 & 0 & 0 & 0 & 0 & 0 & 0 & 0 & 0 & 0 & 0 & 0 & 0 & 0 & 0 & 0 & 0 & 0 & 0 & 0 & 0 & 0 & 0 & 0 & 1 & 1 & 1 & 1 \\
\hline & Wing tips obtuse & 0 & 0 & 1 & 0 & 0 & 0 & 0 & 0 & 0 & 0 & 0 & 0 & 1 & 1 & 1 & 1 & 1 & 1 & 1 & 1 & 1 & 0 & 0 & 0 & 0 & 0 & 0 & 0 & 0 & 0 & 0 & 0 & 0 & 0 & 0 & 0 \\
\hline
\end{tabular}




\begin{tabular}{|c|c|c|c|c|c|c|c|c|c|c|c|c|c|c|c|c|c|c|c|c|c|c|c|c|c|c|c|c|c|c|c|c|c|c|c|c|c|}
\hline 124 & $\begin{array}{l}\text { Keel tips obtuse } \\
\text { Stamens: } \\
\text { Filaments: }\end{array}$ & 1 & 0 & 1 & 0 & 0 & 0 & 0 & 1 & 1 & 0 & 0 & 0 & 1 & 1 & 1 & 1 & 1 & 0 & 0 & 0 & 0 & 0 & 0 & 0 & 0 & 0 & 0 & 0 & 0 & 0 & 0 & 0 & 0 & 0 & 0 & 0 \\
\hline 125 & Free & 0 & 0 & 0 & 1 & 1 & 1 & 0 & 1 & 1 & 0 & 0 & 0 & 0 & 0 & 0 & 0 & 0 & 0 & 0 & 0 & 0 & 0 & 0 & 0 & 0 & 0 & 0 & 0 & 0 & 0 & 0 & 0 & 0 & 0 & 0 & 0 \\
\hline 126 & Monadelphous & 1 & 1 & 0 & 0 & 0 & 0 & 0 & 0 & 0 & 0 & 0 & 0 & 0 & 0 & 0 & 0 & 0 & 0 & 0 & 0 & 0 & 0 & 0 & 0 & 0 & 0 & 0 & 0 & 0 & 0 & 0 & 0 & 0 & 0 & 0 & 0 \\
\hline 127 & $\begin{array}{l}\text { Diadelphous } \\
\text { Anthers: }\end{array}$ & 0 & 0 & 1 & 0 & 0 & 0 & 1 & 0 & 0 & 1 & 1 & 1 & 1 & 1 & 1 & 1 & 1 & 1 & 1 & 1 & 1 & 1 & 1 & 1 & 1 & 1 & 1 & 1 & 1 & 1 & 1 & 1 & 1 & & 1 & 1 \\
\hline 128 & Ellipsoid to ovoid & 0 & 0 & 0 & 0 & 0 & 0 & 0 & 0 & 0 & 0 & 0 & 0 & 0 & 0 & 0 & 0 & 0 & 0 & 0 & 0 & 0 & 0 & 0 & 0 & 0 & 0 & 0 & 0 & 0 & 0 & 0 & 0 & 1 & 1 & 1 & 1 \\
\hline 129 & Uniform & 1 & 1 & 1 & 1 & 1 & 1 & 1 & 1 & 1 & 0 & 0 & 0 & 1 & 1 & 1 & 1 & 1 & 1 & 1 & 1 & 1 & 1 & 1 & 1 & 1 & 1 & 1 & 1 & 1 & & 1 & 1 & 0 & & 0 & 0 \\
\hline 130 & $\begin{array}{l}\text { Dorsifixed } \\
\text { Pod: }\end{array}$ & 0 & 0 & 0 & 0 & 0 & 0 & 0 & 0 & 0 & 1 & 1 & 1 & 0 & 0 & 0 & 0 & 0 & 0 & 0 & 0 & 0 & 0 & 0 & 0 & 0 & 0 & 0 & 0 & 0 & 0 & 0 & 0 & 0 & 0 & 0 & 0 \\
\hline 131 & $\geq 0.1-0.8 \mathrm{~cm}$ & 1 & 1 & 0 & 0 & 0 & 0 & 0 & 0 & 0 & 0 & 0 & 0 & 0 & 1 & 0 & 0 & 0 & 1 & 1 & 1 & 1 & 1 & 1 & 1 & 1 & 1 & 1 & 1 & 1 & & 1 & & 0 & & 0 & 0 \\
\hline 132 & $<0.8-3 \mathrm{~cm}$ & 0 & 0 & 0 & 1 & 1 & 1 & 1 & 1 & 1 & 1 & 1 & 1 & 1 & 0 & 1 & 1 & 1 & 0 & 0 & 0 & 0 & 0 & U & 0 & 0 & 0 & 0 & 0 & 0 & 0 & 0 & 0 & 0 & & 1 & 1 \\
\hline 133 & $\begin{array}{l}<3-9 \mathrm{~cm} \\
\text { Pod Shape: }\end{array}$ & 0 & 0 & 1 & 0 & 0 & 0 & 0 & 0 & 0 & 0 & 0 & 0 & 0 & 0 & 0 & 0 & 0 & 0 & 0 & 0 & 0 & 0 & 0 & 0 & 0 & 0 & 0 & 0 & 0 & 0 & 0 & 0 & 1 & 1 & 0 & 0 \\
\hline 134 & linear-oblong & 0 & 0 & 0 & 0 & 0 & 1 & 1 & 1 & 1 & 0 & 0 & 0 & 0 & 0 & 0 & 0 & 0 & 0 & 0 & 0 & 0 & 0 & 0 & 0 & 0 & 0 & 0 & 0 & 0 & 0 & 0 & Q & 0 & 0 & 0 & 0 \\
\hline 135 & Oblong & 0 & 0 & 1 & 1 & 1 & 0 & 0 & 0 & 0 & 0 & 0 & 0 & 0 & 0 & 0 & 0 & 0 & 0 & 0 & 0 & 0 & 0 & 0 & 0 & 0 & 0 & 0 & 0 & 0 & 0 & 0 & & 0 & & 0 & 0 \\
\hline 136 & Ovate & 1 & 0 & 0 & 0 & 0 & 0 & 0 & 0 & 0 & 0 & 0 & 0 & 0 & 0 & 0 & 0 & 0 & 1 & 1 & 0 & 1 & 1 & 1 & 1 & 1 & 1 & 1 & 1 & 1 & 1 & 1 & 1 & 1 & & 1 & 1 \\
\hline 137 & Slightly curved & 0 & 1 & 0 & 0 & 0 & 0 & 1 & 0 & 0 & 0 & 0 & 0 & 1 & 1 & 1 & 0 & 1 & 0 & 0 & 0 & 0 & 0 & 0 & 0 & 0 & 0 & 0 & 0 & 0 & 0 & 0 & 0 & 0 & & 0 & 0 \\
\hline 138 & Spirally twisted & 0 & 0 & 0 & 0 & 0 & 0 & 0 & 0 & 0 & 0 & 0 & 0 & 0 & 0 & 0 & 1 & 0 & 0 & 0 & 0 & 0 & 0 & 0 & 0 & 0 & 0 & 0 & 0 & 0 & 0 & 0 & 0 & 0 & 0 & 0 & 0 \\
\hline 139 & Subglobose & 0 & 0 & 0 & 0 & 0 & 0 & 0 & 0 & 0 & 1 & 1 & 0 & 0 & 0 & 0 & 0 & 0 & 0 & 0 & 1 & 0 & 0 & 0 & 0 & 0 & 0 & 0 & 0 & 0 & 0 & 0 & 0 & 0 & & 0 & 0 \\
\hline 140 & Flat & 0 & 0 & 0 & 0 & 0 & 0 & 0 & 1 & 1 & 0 & 0 & 0 & 0 & 0 & 0 & 0 & 0 & 0 & 0 & 0 & 0 & 0 & 0 & 0 & 0 & 0 & 0 & 0 & 0 & 0 & 0 & 0 & 0 & & 0 & 0 \\
\hline 141 & Inflated & 0 & 0 & 1 & 0 & 0 & 0 & 0 & 0 & 0 & 0 & 0 & 0 & 0 & 0 & 0 & 0 & 0 & 0 & 0 & 0 & 0 & 0 & 0 & 0 & 0 & 0 & 0 & 0 & 0 & 0 & 0 & 0 & 0 & & 0 & 0 \\
\hline 142 & Hooked & 0 & 1 & 0 & 0 & 0 & 0 & 0 & 0 & 0 & 0 & 0 & 0 & 0 & 0 & 0 & 0 & 0 & 0 & 0 & 0 & 0 & 0 & 0 & 0 & 0 & 0 & 0 & 0 & 0 & 0 & 0 & 0 & 0 & 0 & 0 & 0 \\
\hline 143 & sinus almost closed & 0 & 0 & 0 & 0 & 0 & 0 & 1 & 0 & 0 & 0 & 0 & 0 & 0 & 0 & 0 & 0 & 0 & 0 & 0 & 0 & 0 & 0 & 0 & 0 & 0 & 0 & 0 & 0 & 0 & 0 & 0 & 0 & 0 & & 0 & 0 \\
\hline 144 & Straight & 0 & 0 & 0 & 0 & 0 & 0 & 0 & 0 & 0 & 0 & 0 & 1 & 1 & 1 & 1 & 1 & 1 & 0 & 0 & 0 & 0 & 0 & 0 & 0 & 0 & 0 & 0 & 0 & 0 & 0 & 0 & 0 & 0 & & 0 & 0 \\
\hline 145 & $\begin{array}{l}\text { Terete } \\
\text { Pod Wall: }\end{array}$ & 0 & 0 & 0 & 0 & 0 & 0 & 0 & 0 & 0 & 0 & 0 & 1 & 0 & 0 & 0 & 0 & 0 & 0 & 0 & 0 & 0 & 0 & 0 & 0 & 0 & 0 & 0 & 0 & 0 & 0 & 0 & 0 & 0 & U & 0 & 0 \\
\hline 146 & $\begin{array}{l}\text { thick-walled } \\
\text { Pod Type: }\end{array}$ & 0 & 0 & 0 & 0 & 0 & 0 & 0 & 1 & 1 & 0 & 0 & 0 & 1 & 1 & 1 & 1 & 1 & 1 & 1 & 1 & 1 & 0 & 0 & 0 & 0 & 0 & 0 & 0 & 0 & 0 & 0 & 0 & 0 & U & 0 & 0 \\
\hline 147 & Indehiscent & 1 & 1 & 0 & 0 & 0 & 0 & 0 & 0 & 0 & 0 & 0 & 0 & 1 & 1 & 1 & 1 & 1 & 0 & 0 & 0 & 0 & 1 & 1 & 1 & 1 & 1 & 1 & 1 & 1 & 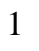 & 1 & & 0 & & 0 & 0 \\
\hline 148 & $\begin{array}{l}\text { Dehiscent } \\
\text { Pod Apex: }\end{array}$ & 0 & 0 & 1 & 1 & 1 & 1 & 1 & 1 & 1 & 1 & 1 & 1 & 0 & 0 & 0 & 0 & 0 & 1 & 1 & 1 & 1 & 0 & 0 & 0 & 0 & 0 & 0 & 0 & 0 & 0 & 0 & 0 & 1 & 1 & 1 & 1 \\
\hline 149 & Acute & 1 & 0 & 1 & 1 & 1 & 0 & 0 & 1 & 1 & 1 & 1 & 1 & 0 & 0 & 0 & 0 & 0 & 0 & 0 & 0 & 0 & 0 & & 0 & 0 & 0 & 0 & 0 & 0 & 0 & 0 & & 0 & & 0 & 0 \\
\hline 15 & Beaked & 0 & 0 & 0 & 0 & 0 & 0 & 0 & 0 & 0 & 0 & 0 & 0 & 1 & 1 & 1 & 1 & 1 & 1 & 1 & 1 & 1 & 1 & 1 & 1 & 1 & 1 & 1 & 1 & 1 & 1 & 1 & & 0 & & 0 & 0 \\
\hline & Obtuse & 0 & 0 & 0 & 0 & 0 & 1 & 1 & 0 & 0 & 0 & 0 & 0 & 0 & 0 & 0 & 0 & 0 & 0 & 0 & 0 & 0 & 0 & 0 & 0 & 0 & 0 & 0 & 0 & 0 & 0 & 0 & 0 & 0 & & 0 & 0 \\
\hline
\end{tabular}


Pod Surface:

\begin{tabular}{l|l}
152 & Hairy \\
153 & Veined \\
154 & Pubescent \\
155 & Smooth \\
156 & Pilose \\
157 & Pod Compression: \\
Compressed
\end{tabular}

157 Compressed

158 Deflexed

Pod Segment

number:

$159 \geq 6$

$160<7-12$

$161<12$

Seed

Compression:

162 Compressed

163 Not compressed

Seed Shape:

164 Cylindrical

165 Flattened

166 Ovoid

167 Reniform

Seed Surface:

168 Fine tuberculate

169 Finely reticulate

170 Glossy

171 Seeds Number:

$171 \geq 4$

$172<4-8$

$173<8-15$

\section{Mr. (kDa)}

$174 \quad 117.71$

$175 \quad 115.85$

$1 7 6 \longdiv { 1 1 1 . 0 0 }$ $\begin{array}{llllllllllllllllllllllllllllllllllllll}0 & 1 & 1 & 1 & 1 & 1 & 0 & 0 & 0 & 0 & 0 & 0 & 0 & 0 & 0 & 0 & 0 & 0 & 0 & 0 & 0 & 0 & 0 & 0 & 0 & 0 & 0 & 0 & 0 & 0 & 0 & 0 & 0 & 0 & 0 & 0\end{array}$

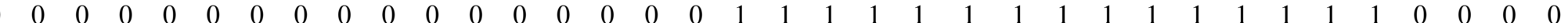
$\begin{array}{llllllllllllllllllllllllllllllllllllllllllllllll}1 & 0 & 0 & 0 & 0 & 0 & 0 & 0 & 1 & 0 & 0 & 0 & 1 & 0 & 0 & 0 & 0 & 0 & 0 & 0 & 0 & 0 & 0 & 0 & 0 & 0 & 0 & 0 & 0 & 0 & 0 & 0 & 0 & 0 & 0 & 0\end{array}$

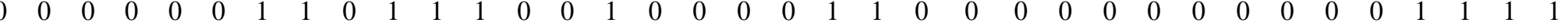

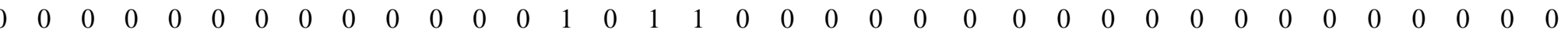
\begin{tabular}{llllllllllllllllllllllllllllllllllllllllll}
1 & 0 & 1 & 1 & 1 & 1 & 1 & 1 & 1 & 1 & 1 & 1 & 1 & 1 & 1 & 1 & 1 & 1 & 1 & 1 & 1 & 1 & 1 & 1 & 1 & 1 & 1 & 1 & 1 & 1 & 1 & 1 & 1 & 1 & 1 & 1 \\
\hline
\end{tabular} $\begin{array}{llllllllllllllllllllllllllllllllllllllllllllll}0 & 1 & 0 & 0 & 0 & 0 & 0 & 0 & 0 & 0 & 0 & 0 & 0 & 0 & 0 & 0 & 0 & 0 & 0 & 0 & 0 & 0 & 0 & 0 & 0 & 0 & 0 & 0 & 0 & 0 & 0 & 0 & 0 & 0 & 0 & 0\end{array}$ $\begin{array}{lllllllllllllllllllllllllllllllllllllllllllll}1 & 1 & 1 & 1 & 1 & 1 & 1 & 1 & 0 & 0 & 0 & 0 & 1 & 1 & 1 & 1 & 1 & 1 & 1 & 1 & 1 & 1 & 1 & 1 & 1 & 1 & 1 & 1 & 1 & 1 & 1 & 1 & 0 & 1 & 1 & 1 \\ & 0 & 0 & 0 & 0 & 0 & 0 & 0 & 1 & 0 & 0 & 0 & 0 & 0 & 0 & 0 & 0 & 0 & 0 & 0 & 0 & 0 & 0 & 0 & 0 & 0 & 0 & 0 & 0 & 0 & 0 & 0 & 1 & 0 & 0 & 0\end{array}$ \begin{tabular}{llllllllllllllllllllllllllllllllllllllllllll}
0 & 0 & 0 & 0 & 0 & 0 & 0 & 0 & 1 & 0 & 0 & 0 & 0 & 0 & 0 & 0 & 0 & 0 & 0 & 0 & 0 & 0 & 0 & 0 & 0 & 0 & 0 & 0 & 0 & 0 & 0 & 0 & 1 & 0 & 0 & 0 \\
\hline
\end{tabular}

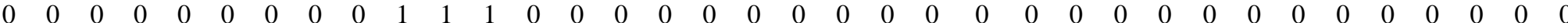

$\begin{array}{lllllllllllllllllllllllllllllllllllllllllll}1 & 0 & 1 & 1 & 1 & 0 & 1 & 0 & 0 & 0 & 0 & 0 & 1 & 1 & 1 & 1 & 1 & 1 & 1 & 1 & 1 & 1 & 1 & 1 & 1 & 1 & 1 & 1 & 1 & 1 & 1 & 1 & 1 & 1 & 1 & 1\end{array}$

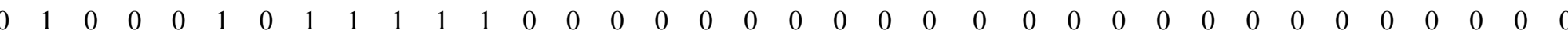
$\begin{array}{llllllllllllllllllllllllllllllllllllllllllll}0 & 1 & 0 & 1 & 1 & 0 & 1 & 0 & 0 & 1 & 1 & 1 & 0 & 0 & 0 & 0 & 0 & 0 & 0 & 0 & 0 & 0 & 0 & 0 & 0 & 0 & 0 & 0 & 0 & 0 & 0 & 0 & 0 & 0 & 0 & 0\end{array}$ $\begin{array}{llllllllllllllllllllllllllllllllllllllllll}0 & 0 & 0 & 0 & 0 & 0 & 0 & 1 & 1 & 0 & 0 & 0 & 0 & 0 & 0 & 0 & 0 & 0 & 0 & 0 & 0 & 0 & 0 & 0 & 0 & 0 & 0 & 0 & 0 & 0 & 0 & 0 & 0 & 1 & 1 & 1\end{array}$ $\begin{array}{llllllllllllllllllllllllllllllllllllllllllllllll}1 & 0 & 0 & 0 & 0 & 1 & 0 & 1 & 1 & 0 & 0 & 0 & 0 & 0 & 1 & 0 & 1 & 1 & 1 & 1 & 1 & 1 & 1 & 1 & 1 & 1 & 0 & 1 & 1 & 1 & 1 & 1 & 1 & 1 & 1 & 1\end{array}$ $\begin{array}{llllllllllllllllllllllllllllllllllllllllllllll}0 & 0 & 1 & 0 & 0 & 0 & 0 & 0 & 0 & 0 & 0 & 0 & 1 & 1 & 0 & 1 & 0 & 0 & 0 & 0 & 0 & 0 & 0 & 0 & 0 & 0 & 1 & 0 & 0 & 0 & 0 & 0 & 0 & 0 & 0 & 0\end{array}$ $\begin{array}{llllllllllllllllllllllllllllllllllllllll}0 & 0 & 0 & 0 & 0 & 0 & 0 & 0 & 0 & 0 & 0 & 0 & 0 & 0 & 1 & 0 & 0 & 0 & 1 & 1 & 1 & 0 & 0 & 0 & 0 & 0 & 0 & 0 & 0 & 0 & 0 & 0 & 0 & 0 & 0 & 0 \\ 0 & 0 & 0 & 0 & 0 & 0 & 0 & 0 & 0 & 0 & 0 & 0 & 0 & 1 & 0 & 0 & 1 & 0 & 0 & 0 & 0 & 0 & 0 & 0 & 0 & 0 & 0 & 0 & 0 & 0 & 0 & 0 & 0 & 0 & 0 & 0\end{array}$

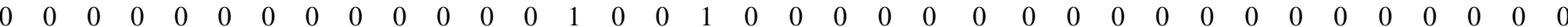

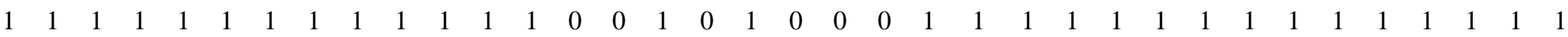

\begin{tabular}{llllllllllllllllllllllllllllllllllllllllllllll}
1 & 1 & 0 & 1 & 1 & 1 & 1 & 0 & 0 & 0 & 0 & 0 & 1 & 1 & 0 & 1 & 1 & 1 & 1 & 1 & 1 & 1 & 1 & 1 & 1 & 1 & 1 & 1 & 1 & 1 & 1 & 1 & 0 & 1 & 0 & 0 \\
\hline
\end{tabular} $\begin{array}{llllllllllllllllllllllllllllllllllllllllll}0 & 0 & 1 & 0 & 0 & 0 & 0 & 1 & 0 & 0 & 0 & 0 & 0 & 0 & 1 & 0 & 0 & 0 & 0 & 0 & 0 & 0 & 0 & 0 & 0 & 0 & 0 & 0 & 0 & 0 & 0 & 0 & 1 & 0 & 1 & 1\end{array}$ \begin{tabular}{llllllllllllllllllllllllllllllllllllll}
0 & 0 & 0 & 0 & 0 & 0 & 0 & 0 & 1 & 1 & 1 & 1 & 0 & 0 & 0 & 0 & 0 & 0 & 0 & 0 & 0 & 0 & 0 & 0 & 0 & 0 & 0 & 0 & 0 & 0 & 0 & 0 & 0 & 0 & 0 & 0 \\
\hline
\end{tabular} Protein Characters*

$\begin{array}{lllllllllllllllllllllllllllllllllllllllllll}0 & 0 & 0 & 0 & 0 & 0 & 0 & 0 & 0 & 0 & 0 & 0 & 0 & 0 & 0 & 0 & 0 & 0 & 0 & 0 & 0 & 0 & 0 & 0 & 1 & 1 & 1 & 1 & 1 & 1 & 1 & 1 & 1 & 0 & 0 & 0 \\ 0 & 0 & 0 & 0 & 0 & 0 & 0 & 0 & 0 & 0 & 0 & 0 & 1 & 1 & 1 & 1 & 1 & 1 & 1 & 1 & 1 & 0 & 1 & 1 & 0 & 0 & 0 & 0 & 0 & 0 & 0 & 0 & 0 & 0 & 0 & 0\end{array}$ $\begin{array}{llllllllllllllllllllllllllllllllllllllllllllll}0 & 0 & 0 & 0 & 0 & 0 & 0 & 0 & 0 & 0 & 0 & 0 & 1 & 1 & 1 & 1 & 1 & 1 & 1 & 1 & 1 & 0 & 1 & 1 & 0 & 0 & 0 & 0 & 0 & 0 & 0 & 0 & 0 & 0 & 0 & 0\end{array}$

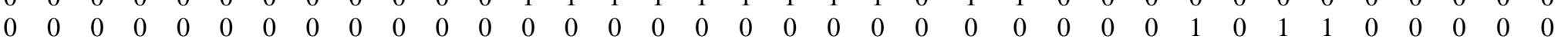




\begin{tabular}{|l|l|}
177 & 108.00 \\
178 & 106.41 \\
179 & 105.10 \\
180 & 102.97 \\
181 & 100.91 \\
182 & 99.00 \\
183 & 98.65 \\
184 & 97.66 \\
185 & 95.00 \\
186 & 90.16 \\
187 & 87.39 \\
188 & 85.76 \\
189 & 83.93 \\
190 & 81.41 \\
191 & 80.61 \\
192 & 79.23 \\
193 & 78.00 \\
194 & 76.00 \\
195 & 73.61 \\
196 & 69.00 \\
197 & 68.10 \\
198 & 67.38 \\
199 & 66.54 \\
200 & 65.00 \\
201 & 63.22 \\
202 & 59.00 \\
203 & 56.90 \\
204 & 53.81 \\
205 & 52.00 \\
206 & 51.00 \\
207 & 49.00 \\
208 & 48.13 \\
209 & 47.00 \\
210 & 46.00 \\
211 & 45.69 \\
212 & 44.29 \\
& \\
\hline
\end{tabular}

$\begin{array}{lllllllllllllllllllllllllllllllllllllllll}0 & 0 & 0 & 0 & 0 & 0 & 0 & 0 & 0 & 0 & 0 & 0 & 0 & 1 & 1 & 0 & 1 & 0 & 0 & 0 & 0 & 0 & 0 & 0 & 0 & 0 & 0 & 0 & 1 & 0 & 0 & 0 & 0 & 0 & 0 & 0 \\ 1 & 0 & 1 & 1 & 1 & 0 & 1 & 1 & 1 & 1 & 1 & 1 & 0 & 0 & 0 & 0 & 0 & 0 & 0 & 0 & 0 & 0 & 0 & 0 & 0 & 0 & 0 & 0 & 0 & 0 & 0 & 0 & 0 & 0 & 0 & 0\end{array}$

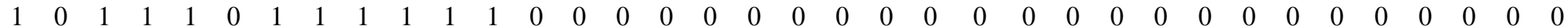

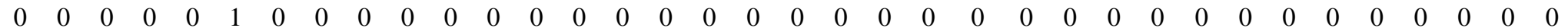
$\begin{array}{llllllllllllllllllllllllllllllllllllllllllllllll}0 & 0 & 0 & 0 & 0 & 0 & 0 & 0 & 0 & 0 & 0 & 0 & 0 & 0 & 0 & 0 & 0 & 0 & 0 & 0 & 0 & 0 & 0 & 0 & 0 & 0 & 0 & 1 & 0 & 1 & 1 & 0 & 0 & 0 & 0 & 0\end{array}$

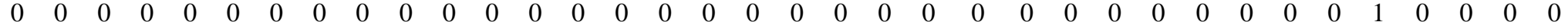
$\begin{array}{lllllllllllllllllllllllllllllllllllllllllllllllll}0 & 0 & 0 & 0 & 0 & 0 & 0 & 0 & 0 & 0 & 0 & 0 & 0 & 1 & 0 & 0 & 0 & 0 & 0 & 0 & 0 & 0 & 1 & 1 & 0 & 0 & 0 & 0 & 0 & 0 & 0 & 0 & 0 & 1 & 0 & 0\end{array}$ $\begin{array}{llllllllllllllllllllllllllllllllllllllllll}0 & 0 & 0 & 0 & 0 & 0 & 0 & 0 & 0 & 0 & 0 & 0 & 0 & 0 & 0 & 0 & 0 & 0 & 0 & 0 & 0 & 0 & 0 & 0 & 0 & 0 & 0 & 0 & 0 & 0 & 0 & 0 & 0 & 0 & 1 & 1\end{array}$ $\begin{array}{llllllllllllllllllllllllllllllllllllllllllllllllll}0 & 0 & 0 & 0 & 0 & 0 & 0 & 0 & 0 & 0 & 0 & 0 & 0 & 0 & 0 & 0 & 0 & 0 & 0 & 0 & 0 & 0 & 0 & 0 & 0 & 0 & 0 & 0 & 0 & 0 & 0 & 0 & 0 & 0 & 1 & 0\end{array}$ $\begin{array}{lllllllllllllllllllllllllllllllllllllllllllllll}0 & 0 & 0 & 0 & 0 & 0 & 0 & 0 & 0 & 0 & 0 & 0 & 0 & 0 & 0 & 0 & 0 & 0 & 0 & 0 & 0 & 0 & 0 & 0 & 0 & 0 & 0 & 0 & 1 & 0 & 0 & 1 & 0 & 0 & 1 & 0\end{array}$ $\begin{array}{lllllllllllllllllllllllllllllllllllllllllllll}0 & 0 & 0 & 0 & 0 & 0 & 0 & 0 & 0 & 0 & 0 & 0 & 0 & 0 & 1 & 0 & 0 & 0 & 0 & 0 & 0 & 0 & 0 & 0 & 0 & 0 & 0 & 0 & 0 & 0 & 0 & 0 & 0 & 0 & 0 & 0\end{array}$

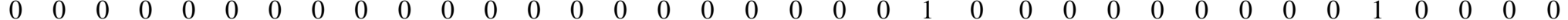
$\begin{array}{llllllllllllllllllllllllllllllllllllllll}0 & 1 & 0 & 0 & 0 & 0 & 0 & 0 & 0 & 0 & 0 & 0 & 0 & 0 & 0 & 0 & 0 & 0 & 0 & 0 & 0 & 0 & 0 & 0 & 0 & 0 & 0 & 0 & 0 & 0 & 0 & 0 & 0 & 0 & 0 & 0\end{array}$ $\begin{array}{lllllllllllllllllllllllllllllllllllllllllll}0 & 0 & 0 & 0 & 0 & 0 & 0 & 0 & 0 & 0 & 0 & 0 & 0 & 0 & 0 & 0 & 0 & 0 & 0 & 0 & 0 & 0 & 0 & 0 & 1 & 1 & 1 & 0 & 0 & 0 & 0 & 0 & 0 & 0 & 0 & 0\end{array}$

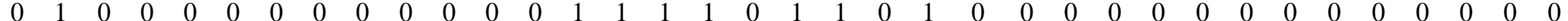
$\begin{array}{llllllllllllllllllllllllllllllllllllllllllllllll}0 & 0 & 0 & 0 & 0 & 0 & 0 & 0 & 0 & 0 & 0 & 0 & 0 & 0 & 0 & 0 & 0 & 0 & 0 & 0 & 0 & 0 & 0 & 0 & 0 & 0 & 0 & 0 & 0 & 0 & 0 & 1 & 0 & 0 & 0 & 0\end{array}$ \begin{tabular}{lllllllllllllllllllllllllllllllllllllllllllll}
0 & 0 & 0 & 0 & 0 & 0 & 0 & 0 & 0 & 0 & 0 & 0 & 0 & 0 & 0 & 0 & 0 & 1 & 0 & 0 & 0 & 0 & 0 & 1 & 0 & 0 & 0 & 0 & 0 & 0 & 0 & 0 & 0 & 0 & 0 & 0 \\
\hline
\end{tabular}

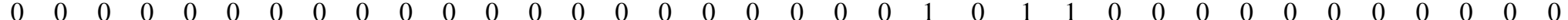

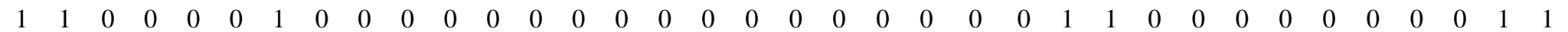
$\begin{array}{llllllllllllllllllllllllllllllllllllllllllllllllll}0 & 0 & 0 & 0 & 0 & 0 & 0 & 0 & 0 & 0 & 0 & 0 & 0 & 0 & 1 & 0 & 0 & 0 & 0 & 0 & 0 & 1 & 0 & 1 & 1 & 0 & 0 & 0 & 0 & 0 & 0 & 0 & 0 & 0 & 0 & 0\end{array}$

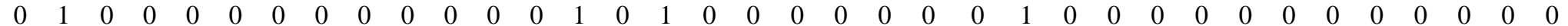

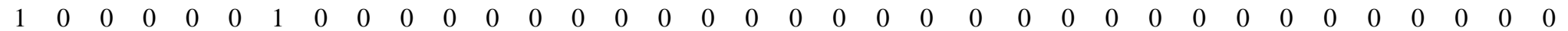
$\begin{array}{llllllllllllllllllllllllllllllllllllllllllllll}0 & 0 & 0 & 0 & 0 & 0 & 0 & 0 & 0 & 0 & 0 & 0 & 0 & 0 & 0 & 0 & 0 & 0 & 0 & 0 & 0 & 0 & 0 & 0 & 0 & 0 & 1 & 1 & 0 & 0 & 0 & 0 & 0 & 0 & 0 & 0\end{array}$

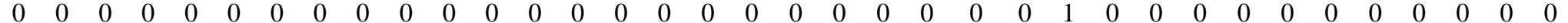
$\begin{array}{lllllllllllllllllllllllllllllllllllllllllll}0 & 0 & 0 & 0 & 0 & 0 & 0 & 0 & 0 & 0 & 0 & 0 & 0 & 1 & 1 & 0 & 0 & 0 & 0 & 0 & 0 & 0 & 0 & 0 & 1 & 0 & 0 & 0 & 0 & 0 & 0 & 0 & 0 & 0 & 0 & 0\end{array}$ $\begin{array}{llllllllllllllllllllllllllllllllllllllllllllllll}0 & 1 & 0 & 0 & 0 & 0 & 0 & 0 & 0 & 1 & 0 & 0 & 0 & 0 & 0 & 0 & 0 & 0 & 0 & 1 & 0 & 0 & 0 & 1 & 0 & 0 & 0 & 0 & 0 & 0 & 0 & 0 & 0 & 0 & 0 & 0\end{array}$

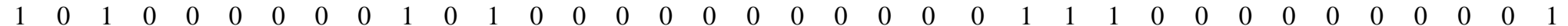
$\begin{array}{lllllllllllllllllllllllllllllllllllllllllllllll}0 & 0 & 0 & 0 & 0 & 0 & 0 & 0 & 0 & 0 & 0 & 0 & 0 & 0 & 0 & 0 & 0 & 0 & 0 & 0 & 0 & 1 & 0 & 0 & 0 & 0 & 0 & 0 & 0 & 0 & 0 & 0 & 0 & 1 & 1 & 1\end{array}$ $\begin{array}{llllllllllllllllllllllllllllllllllllll}0 & 0 & 0 & 0 & 0 & 0 & 1 & 0 & 0 & 0 & 0 & 0 & 0 & 0 & 0 & 0 & 0 & 0 & 0 & 0 & 0 & 0 & 0 & 0 & 0 & 0 & 0 & 0 & 0 & 0 & 0 & 0 & 0 & 0 & 0 & 0\end{array}$

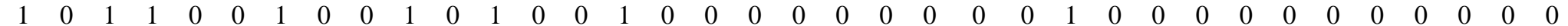
$\begin{array}{llllllllllllllllllllllllllllllllllllllll}1 & 0 & 0 & 0 & 0 & 0 & 0 & 0 & 0 & 0 & 0 & 0 & 0 & 0 & 0 & 0 & 0 & 1 & 0 & 0 & 0 & 0 & 0 & 0 & 0 & 0 & 0 & 0 & 0 & 0 & 0 & 0 & 0 & 0 & 0 & 1\end{array}$ $\begin{array}{llllllllllllllllllllllllllllllllllllllllllllllllll}0 & 0 & 0 & 0 & 0 & 0 & 0 & 0 & 0 & 0 & 0 & 0 & 1 & 1 & 1 & 0 & 0 & 0 & 0 & 0 & 0 & 1 & 0 & 0 & 0 & 0 & 0 & 0 & 0 & 0 & 0 & 0 & 0 & 0 & 0 & 0\end{array}$

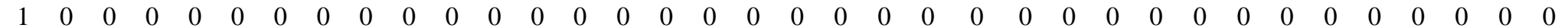
$\begin{array}{llllllllllllllllllllllllllllllllllllllllllllll}0 & 0 & 0 & 0 & 0 & 0 & 0 & 0 & 0 & 0 & 0 & 0 & 0 & 0 & 1 & 0 & 0 & 0 & 1 & 1 & 0 & 0 & 1 & 1 & 0 & 0 & 0 & 0 & 0 & 0 & 0 & 0 & 0 & 0 & 0 & 0\end{array}$

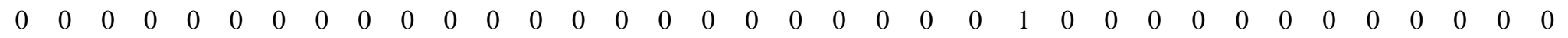

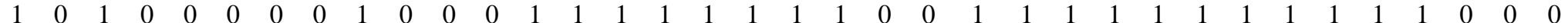

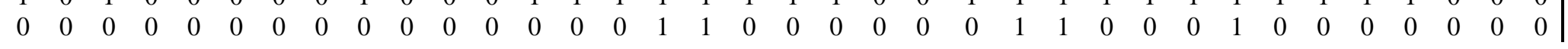




\begin{tabular}{|l|l}
213 & 42.94 \\
214 & 41.74 \\
215 & 40.88 \\
216 & 39.37 \\
217 & 38.48 \\
218 & 37.71 \\
219 & 37.44 \\
220 & 36.55 \\
221 & 35.40 \\
222 & 35.00 \\
223 & 34.52 \\
224 & 33.59 \\
225 & 32.45 \\
226 & 32.00 \\
227 & 31.41 \\
228 & 31.00 \\
229 & 30.74 \\
230 & 30.00 \\
231 & 29.52 \\
232 & 28.74 \\
233 & 27.71 \\
234 & 27.00 \\
235 & 26.62 \\
236 & 26.00 \\
237 & 25.89 \\
238 & 25.00 \\
239 & 24.67 \\
240 & 24.00 \\
241 & 23.68 \\
242 & 23.30 \\
243 & 22.42 \\
244 & 21.50 \\
245 & 21.00 \\
246 & 20.66 \\
247 & 19.56 \\
248 & 19.00 \\
& \\
\hline
\end{tabular}

$\begin{array}{llllllllllllllllllllllllllllllllllllllll}0 & 0 & 0 & 0 & 0 & 0 & 0 & 0 & 0 & 0 & 0 & 0 & 0 & 1 & 0 & 0 & 0 & 0 & 0 & 0 & 0 & 0 & 0 & 0 & 0 & 0 & 0 & 0 & 0 & 0 & 0 & 0 & 1 & 0 & 0 & 0 \\ 0 & 0 & 0 & 0 & 0 & 0 & 0 & 0 & 0 & 0 & 0 & 0 & 0 & 1 & 0 & 0 & 0 & 0 & 0 & 0 & 0 & 0 & 0 & 0 & 0 & 1 & 1 & 0 & 0 & 0 & 0 & 0 & 0 & 0 & 0 & 0\end{array}$

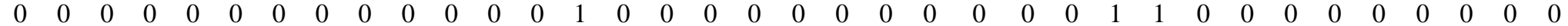

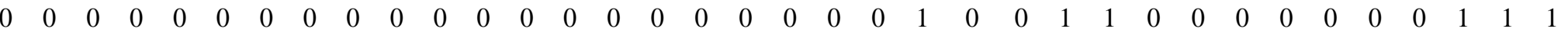
$\begin{array}{lllllllllllllllllllllllllllllllllllll}0 & 0 & 1 & 0 & 0 & 0 & 0 & 1 & 0 & 0 & 0 & 0 & 0 & 0 & 0 & 0 & 0 & 1 & 0 & 0 & 0 & 0 & 0 & 0 & 1 & 0 & 0 & 0 & 0 & 0 & 0 & 0 & 0 & 0 & 0 & 0\end{array}$

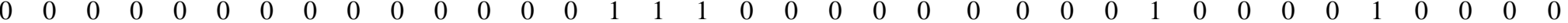

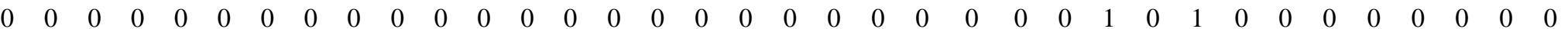

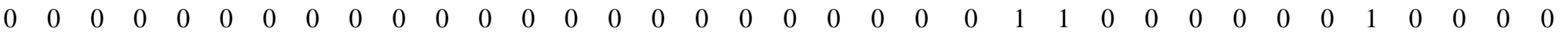

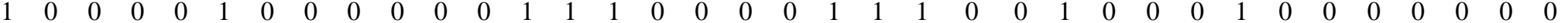

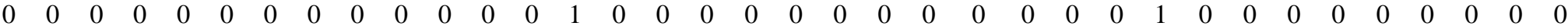
$\begin{array}{llllllllllllllllllllllllllllllllllllllllllllll}0 & 0 & 0 & 0 & 0 & 0 & 0 & 0 & 0 & 0 & 0 & 0 & 1 & 1 & 1 & 1 & 1 & 0 & 0 & 0 & 0 & 0 & 0 & 0 & 0 & 0 & 0 & 0 & 1 & 0 & 0 & 0 & 0 & 0 & 0 & 0\end{array}$

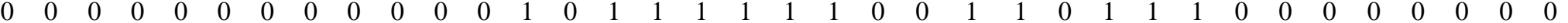
\begin{tabular}{lllllllllllllllllllllllllllllllllllllllllllllllll}
0 & 0 & 0 & 0 & 0 & 0 & 0 & 0 & 0 & 0 & 0 & 0 & 0 & 1 & 0 & 0 & 1 & 0 & 0 & 0 & 0 & 0 & 0 & 0 & 1 & 0 & 0 & 0 & 0 & 0 & 0 & 0 & 0 & 0 & 0 & 0 \\
\hline
\end{tabular}

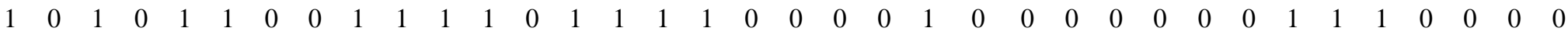

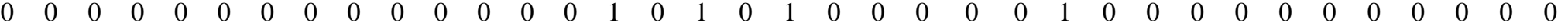

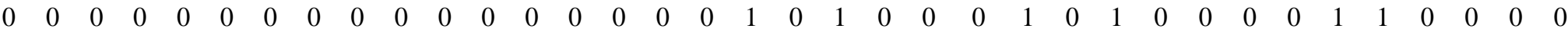
$\begin{array}{llllllllllllllllllllllllllllllllllllllllllllll}0 & 0 & 0 & 0 & 0 & 0 & 0 & 0 & 0 & 0 & 0 & 0 & 0 & 1 & 1 & 1 & 1 & 0 & 0 & 0 & 0 & 0 & 0 & 1 & 1 & 0 & 0 & 0 & 0 & 0 & 0 & 0 & 0 & 0 & 0 & 0\end{array}$

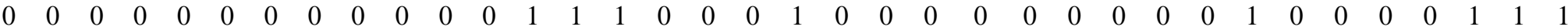

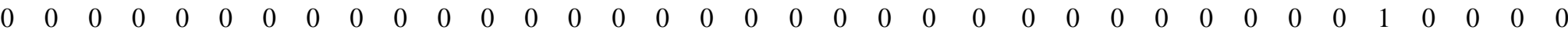
$\begin{array}{lllllllllllllllllllllllllllllllllllllllllllllll}0 & 0 & 0 & 0 & 0 & 0 & 0 & 0 & 0 & 0 & 0 & 0 & 1 & 0 & 1 & 0 & 0 & 0 & 0 & 0 & 0 & 0 & 0 & 1 & 0 & 0 & 0 & 0 & 0 & 0 & 0 & 0 & 1 & 1 & 0 & 0\end{array}$

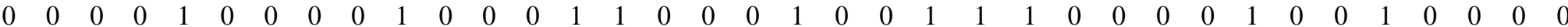

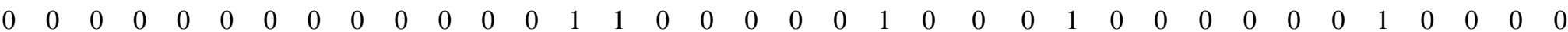
$\begin{array}{lllllllllllllllllllllllllllllllllllllllllll}0 & 0 & 0 & 0 & 0 & 0 & 0 & 0 & 0 & 0 & 0 & 0 & 0 & 0 & 0 & 0 & 1 & 0 & 1 & 0 & 0 & 0 & 0 & 0 & 0 & 0 & 0 & 1 & 0 & 0 & 0 & 0 & 1 & 0 & 0 & 0\end{array}$

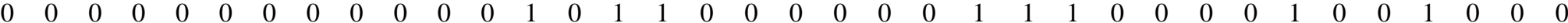

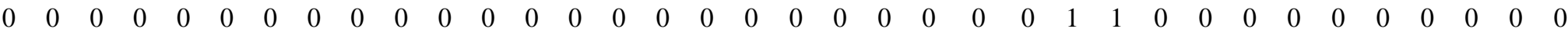
$\begin{array}{llllllllllllllllllllllllllllllllllllllllllllllllll}0 & 0 & 0 & 0 & 0 & 0 & 0 & 0 & 1 & 0 & 0 & 0 & 1 & 1 & 0 & 0 & 0 & 0 & 0 & 0 & 0 & 1 & 1 & 1 & 1 & 1 & 0 & 0 & 0 & 1 & 0 & 0 & 0 & 0 & 0 & 0\end{array}$

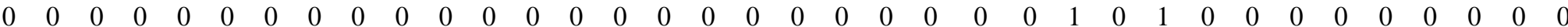
$\begin{array}{llllllllllllllllllllllllllllllllllllllllllllll}0 & 0 & 0 & 0 & 0 & 0 & 0 & 0 & 0 & 0 & 0 & 0 & 0 & 0 & 0 & 0 & 0 & 0 & 0 & 0 & 0 & 0 & 1 & 1 & 1 & 0 & 0 & 0 & 0 & 0 & 0 & 0 & 1 & 1 & 1 & 1\end{array}$ \begin{tabular}{llllllllllllllllllllllllllllllllllllllllllllllll}
0 & 0 & 0 & 0 & 0 & 0 & 0 & 0 & 0 & 0 & 0 & 0 & 0 & 0 & 0 & 0 & 0 & 0 & 0 & 0 & 0 & 0 & 0 & 0 & 1 & 0 & 0 & 0 & 1 & 0 & 0 & 0 & 0 & 0 & 0 & 0 \\
\hline
\end{tabular}

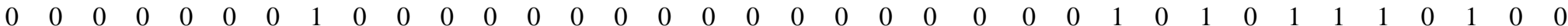
$\begin{array}{lllllllllllllllllllllllllllllllllllllllllllllll}0 & 0 & 0 & 0 & 0 & 0 & 0 & 0 & 0 & 0 & 0 & 0 & 0 & 0 & 0 & 0 & 0 & 0 & 0 & 0 & 0 & 0 & 0 & 0 & 1 & 0 & 1 & 0 & 0 & 0 & 0 & 0 & 1 & 0 & 0 & 0\end{array}$ $\begin{array}{lllllllllllllllllllllllllllllllllllllllllllllll}0 & 0 & 0 & 0 & 0 & 0 & 0 & 0 & 0 & 0 & 0 & 0 & 0 & 0 & 0 & 0 & 0 & 0 & 0 & 0 & 0 & 0 & 0 & 0 & 0 & 1 & 0 & 0 & 1 & 0 & 0 & 0 & 0 & 1 & 1 & 1\end{array}$

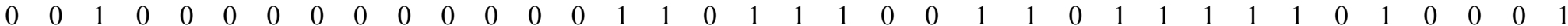
\begin{tabular}{llllllllllllllllllllllllllllllllllllllllllllllll}
0 & 0 & 0 & 0 & 0 & 0 & 0 & 0 & 0 & 0 & 0 & 0 & 0 & 0 & 0 & 0 & 0 & 0 & 0 & 0 & 0 & 0 & 0 & 0 & 0 & 1 & 0 & 0 & 0 & 0 & 0 & 0 & 0 & 0 & 0 & 0 \\
\hline
\end{tabular}

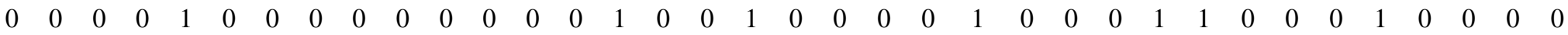

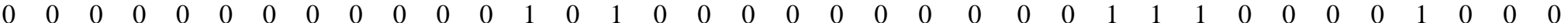
$\begin{array}{lllllllllllllllllllllllllllllllllllllllllllllll}0 & 0 & 0 & 0 & 0 & 0 & 0 & 0 & 0 & 0 & 0 & 0 & 0 & 0 & 0 & 0 & 0 & 0 & 0 & 0 & 0 & 0 & 0 & 0 & 0 & 0 & 1 & 0 & 0 & 0 & 1 & 0 & 0 & 1 & 1 & 1 & 1\end{array}$ 


\begin{tabular}{|c|c|c|c|c|c|c|c|c|c|c|c|c|c|c|c|c|c|c|c|c|c|c|c|c|c|c|c|c|c|c|c|c|c|c|c|c|c|}
\hline 249 & 18.85 & 0 & 0 & 0 & 0 & 0 & 0 & 0 & 0 & 0 & 0 & 0 & 0 & 0 & 1 & 0 & 1 & 0 & 0 & 0 & 0 & 0 & 0 & 0 & 0 & 0 & 0 & 0 & 1 & 1 & 0 & 0 & 0 & 0 & 0 & 0 & 0 \\
\hline 250 & 18.40 & 0 & 0 & 0 & 0 & 0 & 0 & 0 & 0 & 0 & 0 & 0 & 0 & 1 & 1 & 0 & 1 & 0 & 1 & 1 & 0 & 0 & 0 & 0 & 0 & 1 & 0 & 0 & 0 & 0 & 1 & 1 & 0 & 0 & 0 & 0 & 0 \\
\hline 251 & 17.54 & 0 & 0 & 1 & 0 & 0 & 0 & 0 & 0 & 0 & 0 & 0 & 0 & 0 & 0 & 0 & 0 & 0 & 1 & 1 & 0 & 0 & 0 & 0 & 1 & 0 & 1 & 1 & 0 & 0 & 0 & 0 & 0 & 0 & 0 & 0 & 0 \\
\hline 252 & 16.82 & 1 & 0 & 1 & 0 & 1 & 0 & 0 & 1 & 1 & 0 & 1 & 1 & 0 & 0 & 1 & 0 & 0 & 0 & 0 & 1 & 1 & 0 & 0 & 0 & 0 & 0 & 0 & 0 & 0 & 0 & 0 & 0 & 0 & 1 & 1 & 0 \\
\hline 253 & 16.09 & 1 & 0 & 1 & 0 & 1 & 0 & 0 & 0 & 1 & 1 & 1 & 1 & 0 & 0 & 0 & 0 & 0 & 0 & 0 & 0 & 0 & 0 & 0 & 0 & 0 & 0 & 0 & 0 & 0 & 0 & 0 & 0 & 0 & 0 & 0 & 0 \\
\hline 254 & 15.51 & 0 & 0 & 0 & 0 & 0 & 0 & 0 & 0 & 0 & 0 & 0 & 0 & 1 & 1 & 1 & 1 & 1 & 1 & 1 & 1 & 1 & 0 & 1 & 1 & 1 & 0 & 0 & 1 & 1 & 0 & 0 & 0 & 0 & 0 & 0 & 0 \\
\hline 255 & 15.00 & 0 & 0 & 0 & 0 & 0 & 0 & 0 & 0 & 0 & 0 & 0 & 0 & 0 & 0 & 0 & 0 & 0 & 0 & 0 & 0 & 0 & 0 & 1 & 1 & 1 & 0 & 0 & 0 & 0 & 0 & 0 & 0 & 1 & 0 & 0 & 0 \\
\hline 256 & 14.70 & 0 & 0 & 0 & 0 & 0 & 0 & 0 & 0 & 0 & 0 & 0 & 0 & 1 & 0 & 1 & 0 & 0 & 0 & 0 & 0 & 0 & 0 & 0 & 0 & 0 & 1 & 1 & 0 & 1 & 1 & 1 & 1 & 0 & 0 & 0 & 0 \\
\hline 257 & 14.32 & 0 & 0 & 1 & 0 & 0 & 0 & 0 & 0 & 0 & 0 & 0 & 0 & 0 & 0 & 0 & 0 & 0 & 1 & 1 & 1 & 1 & 1 & 1 & 1 & 1 & 1 & 0 & 1 & 0 & 1 & 0 & 0 & 0 & 0 & 0 & 0 \\
\hline 258 & 13.52 & 0 & 0 & 0 & 0 & 0 & 0 & 0 & 0 & 0 & 0 & 0 & 0 & 1 & 0 & 0 & 0 & 0 & 0 & 0 & 1 & 1 & 0 & 0 & 0 & 0 & 0 & 1 & 0 & 1 & 0 & 1 & 1 & 1 & 0 & 0 & 0 \\
\hline 259 & 13.00 & 0 & 0 & 0 & 0 & 0 & 0 & 0 & 0 & 0 & 0 & 0 & 0 & 1 & 0 & 0 & 0 & 0 & 0 & 0 & 0 & 0 & 0 & 0 & 0 & 0 & 0 & 1 & 0 & 0 & 0 & 0 & 0 & 1 & 0 & 0 & 0 \\
\hline 260 & 12.60 & 0 & 0 & 0 & 0 & 0 & 0 & 0 & 0 & 0 & 0 & 0 & 0 & 0 & 0 & 0 & 0 & 0 & 0 & 0 & 1 & 0 & 0 & 0 & 1 & 0 & 0 & 0 & 0 & 0 & 0 & 0 & 0 & 1 & 1 & 1 & 1 \\
\hline 261 & 12.00 & 0 & 0 & 0 & 0 & 0 & 0 & 0 & 0 & 0 & 0 & 0 & 0 & 0 & 1 & 0 & 0 & 0 & 0 & 0 & 0 & 0 & 0 & 0 & 1 & 0 & 0 & 0 & 0 & 0 & 0 & 0 & 0 & 1 & 1 & 1 & 1 \\
\hline 262 & 11.95 & 0 & 0 & 0 & 0 & 0 & 0 & 0 & 0 & 0 & 0 & 0 & 0 & 1 & 1 & 0 & 0 & 0 & 0 & 0 & 0 & 0 & 0 & 0 & 0 & 0 & 0 & 0 & 0 & 0 & 0 & 0 & 0 & 0 & 1 & 1 & 1 \\
\hline 263 & 11.79 & 0 & 0 & 0 & 0 & 0 & 1 & 0 & 0 & 0 & 0 & 0 & 0 & 0 & 0 & 1 & 0 & 0 & 1 & 0 & 1 & 1 & 0 & 0 & 0 & 0 & 0 & 0 & 0 & 0 & 0 & 0 & 0 & 0 & 1 & 0 & 0 \\
\hline 264 & 11.54 & 0 & 0 & 0 & 0 & 0 & 0 & 0 & 0 & 0 & 0 & 0 & 0 & 0 & 0 & 0 & 0 & 0 & 0 & 0 & 0 & 0 & 0 & 1 & 1 & 1 & 0 & 0 & 1 & 0 & 0 & 0 & 0 & 0 & 0 & 0 & 0 \\
\hline 265 & 11.03 & 1 & 0 & 0 & 0 & 0 & 0 & 0 & 0 & 0 & 0 & 0 & 0 & 1 & 1 & 0 & 1 & 0 & 1 & 0 & 0 & 0 & 0 & 0 & 0 & 1 & 0 & 0 & 1 & 0 & 0 & 0 & 0 & 1 & 1 & 1 & 1 \\
\hline 266 & 10.80 & 0 & 0 & 0 & 0 & 0 & 0 & 0 & 0 & 0 & 0 & 0 & 0 & 0 & 0 & 1 & 0 & 0 & 1 & 0 & 1 & 0 & 0 & 0 & 0 & 1 & 1 & 0 & 1 & 1 & 1 & 1 & 1 & 0 & 1 & 1 & 1 \\
\hline 267 & 10.50 & 0 & 0 & 0 & 0 & 0 & 0 & 0 & 0 & 0 & 0 & 0 & 0 & 0 & 0 & 1 & 1 & 0 & 1 & 1 & 0 & 0 & 0 & 0 & 0 & 1 & 0 & 1 & 1 & 1 & 1 & 0 & 0 & 0 & 0 & 0 & 0 \\
\hline 268 & 10.02 & 0 & 0 & 0 & 0 & 0 & 0 & 0 & 0 & 0 & 0 & 0 & 0 & 0 & 0 & 0 & 0 & 0 & 0 & 0 & 0 & 0 & 1 & 0 & 1 & 1 & 1 & 0 & 0 & 0 & 0 & 0 & 0 & 1 & 0 & 0 & 0 \\
\hline 269 & 9.58 & 0 & 0 & 0 & 1 & 1 & 0 & 1 & 0 & 0 & 1 & 1 & 1 & 0 & 0 & 0 & 1 & 0 & 0 & 1 & 1 & 0 & 0 & 1 & 1 & 0 & 0 & 0 & 0 & 0 & 0 & 0 & 0 & 0 & 0 & 0 & 0 \\
\hline 270 & 9.42 & 0 & 0 & 0 & 0 & 0 & 0 & 0 & 0 & 0 & 0 & 0 & 0 & 0 & 0 & 1 & 0 & 0 & 1 & 1 & 0 & 0 & 0 & 0 & 0 & 0 & 0 & 0 & 0 & 0 & 0 & 0 & 0 & 0 & 0 & 0 & 0 \\
\hline
\end{tabular}




\section{References}

1. Greuter, W., Mcneil, J., Barrie, F. R., Burdet, H. M., Demoulin, V., Filqueiras, T. S., Nicolson, D., Silva, P. C., Skog, J. E., \& Hawksworth, D.L. (eds.) (2000). The International Code of Botanical Nomenclature (Saint Louis Code). Koeltz Scientific Books. Konigstein, Germany.

2. Lewis, G. P., Schrire, B. D., MacKinder, B.A. \& Lock, M. (2005). Legumes of the World. Royal Botanic Gardens, 57-67.

3. LPWG, (2013). The Legume Phylogeny Working Group. Legume phylogeny and classification in the 21st century: Progress, prospects and lessons for other species-rich clades. Taxon 62, 217-248.

4. Vargas, E. M., Castro, E., Macaya, G. \& Rocha, O. J. (2003). Variation in fruit and seed size from 38 wild populations of Phaseolus lunatus (Fabaceae) from Central Valley, Costa Rica. Revis. Biol. Trop, 51, 707-724.

5. Singh, K.B., Foley, R.C. \& Onate-Sanchez, L. (2002). Transcription factors in plant defense and stress responses. Curr. Opin. Plant Biol, 5, 430-436.

6. Badr, A. (1995). Electrophoretic studies of seed proteins in relation to chromosomal criteria and the relationships of some taxa of Trifolium. Taxon 44, 183-191.

7. Badr, A., Abou-El-Enain, M. M. \& ElShazly, H. H. (1998). Variation in seed protein electrophoretic pattern and species relationships in Sesbania. Proceedings of the $6^{\text {th }}$ Egyptian Botanical Conference of Plant Sciences, Cairo University (November 24-26, 1998) 3, 493-501. 8. Badr, A., El-Shazly, H. \& Abou-El-Enain, M. M. (2000). Seed protein diversity and its implications on the relationships in the genus Lathyrus L. Proceedings of the $1^{\text {st }}$ International Conference on Biological Sciences, Tanta University, Tanta, Egypt (May 7-8, 2000) 1- 15. 9. Abou-El-Enain, M. M. (2004). SDS-PAGE of seed protein criteria in relation to taxonomy of Onobrychis Sect. Lophobrychis s. str. and the Egyptian species. Cytologia, 69, 351-358.
10. Bijendra, K., Malaviya, D. R., Roy, A. K. \& Kaushal, P. (2003). Protein profile and species relationship in Trifolium. Ind. J. Gen. Pl. Breed, 63, 41-44.

11. Emre, I. (2011). Determination of genetic diversity in the Vicia L. (section vicia) by using SDS-PAGE. Pak. J. Bot, 43(3), 1429-1432.

12. Emre, I., Turgut-Balik, D., Genç, H. \& Şahin, A. (2010). Total seed storage protein patterns of some Lathyrus species growing in turkey using SDS-PAGE. Pak. J. Bot, 42(5), 3157-3163.

13. Cildir, H. (2011). Morphology, anatomy and systematics of the genus Lathyrus. (leguminosae) in central Anatolia, Turkey. A thesis submitted to the graduate school of natural and applied sciences of middle east technical university in partial fulfillment of the requirements for the degree of doctor of philosophy in biology.

14. Doyle, J. J., Doyle, J. L., Ballenger, J. A., Dickson, E. E., Kajita, T. \& Ohashi, H. (1997). A phylogeny of the chloroplast gene rbcL in the Leguminosae: taxonomic correlations and insights into the evolution of nodulation. Amer. J. Bot, 84, 541-554.

15. Wojciechowski, M. F., Lavin, M. \& Sanderson, M. J. (2004). A phylogeny of the legumes (Leguminosae) based on analysis of the plastid matK gene sequences resolves many wellsupported subclades within the family. Amer. J. Bot, 91, 1846-1862.

16. Crisp, M. D., Gilmore, S. \& van-Wyk, B. E. (2000). Molecular phylogeny of the genistoid tribes of papilionoid legumes. In: Herendeen P. S., Bruneau A., eds. Advances in legume systematics part 9. Kew: Royal Botanic Gardens, 249-276.

17. Hu, J. M., Lavin, M., Wojciechowski, M. F. \& Sanderson, M. J. (2000). Phylogenetic analysis of nuclear ribosomal ITS/5.8 S sequences in the tribe Millettieae (Fabaceae): Poecilanthe-Cyclolobium, the core Millettieae and Callerya group. Syst. Bot, 27, 722-733.

18. Pennington, R. T., Prado, D. E. \& Pendry, C. A. (2001). Neotropical seasonally dry forests and Quaternary vegetation changes. J. Biogeogr, 27, 261-273. 
19. Lavin, M., Pennington, R. T., Klitgaard, B. B., Sprent, J. I., de Lima, H. C. \& Gasson, P.E. (2001). The dalbergioid legumes (Fabaceae): delimitation of a pantropical monophyletic clade. Amer. J. Bot, 88, 503-533.

20. Crisp, M. D. \& Cook, L. G. (2003). Molecular evidence for definition of genera in the Oxylobium group (Fabaceae: Mirbelieae). Syst. Bot, 28, 705-713.

21. Boatwright, J. S., Le, A. T., Wink, M. M., Morozova, T. \& van Wyk, B. E. (2008). Phylogenetic relationships of tribe Crotalarieae (Fabaceae) inferred from DNA sequences and morphology. Syst. Bot, 33, 752-761.

22. Egan, A. N. \& Crandall, K. A. (2008). Incorporating gaps as phylogenetic characters across eight DNA regions: Ramifications for North American Psoraleeae (Leguminosae). Molec. Phylogen. Evol, 46, 532-546.

23. Täeckholm, V. (1974). Student's Flora of Egypt. Cairo University. 283-284.

24. Boulos, L. (1999). Flora of Egypt. Al Hadara Publishing, Cairo. 338-339.

25. Ball, P. W. (1968). Papilionoideae. In: Tuten, T.G., Heywood, V. H., Burges, N. A., Moore, D. M., Valentine, S. M. \& Webb, D. A. (Eds.). Flora Europeaea (2), Cambridge University Press.

26. Baily, L. H. \& Bailey, E. Z. (1976). Hortus Third: A concise dictionary of plants cultivated in the United States and Canada. MacMillan Publishing Co., New York. 1290.

27. Laemmli, U. (1970). Cleavage of structural proteins during the assembly of the head of bacteriophage T4. Nature, 227, 680-685.

28. Rohlf, F. J. (2005). NTSYS-pc, Numerical taxonomy and multivariate analysis system. New York: Exeter Computers.

29. Sneath, P. H. \& Sokal, R. R. (1973). Numerical taxonomy: the principles and practice of numerical classification. W. H. Freeman, San Francisco, California, USA.

30. Rohlf, F. J. \& Sokal, R. R. (1981). Comparing numerical taxonomic studies. Syst. Zool, 30, 459 - 490.
31. Diez, M. J. \& Ferguson, I. K. (1996). Studies of the pollen morphology and taxonomy of the tribes Loteae and Coronilleae (Papilionoideae; Leguminoseae). 3, Coronilla L. and related genera and systematic conclusions. Rev. Paleon. Palyn, 94, 239-257

32. Talavera, S. \& Salgueiro, F. S. (1999). Sobre el tratamiento de la tribu Cytiseae Bercht. \& J. Presl (Papilionoideae, Leguminosae) en "Flora Iberica". Anal. Jard. Bot. Madrid, 57, 200-218. 33. Degtjareva, G. V., Vallejo-roman, C. M., Samigullin, T. H., Requena, M. G. \& Sokoloff, D. D. (2012). Phylogenetics of Anthyllis (Leguminosae: Papilionoideae: Loteae): Partial incongruence between nuclear and plastid markers, a long branch problem and implications for morphological evolution. Molec. Phylogen. Evol, 62, 693-707.

34. Sheppard, J. A. (2000). Selection and Testing of Biological Control Agents for Control of French Broom Genista monspessulana (L.) L. Oregon Department of Agriculture. ODA/CDF Final Report.

35. Lane, P., Davies, S., Hall, E. \& Moore, G. (2004). Dorycnium species as alternative forage plants Australian government. A report for the Rural Industries Research and Development Corporation.

36. Sokoloff, D.D., (2003). On system and phylogeny of the tribe Loteae DC. (Leguminosae). Bull. Mosc. Soc. Nat. Biol. Ser. 108, 35-48.

37. Degtjareva, G. V., Samigullin, T. H., Vallejo-roman, C. M. \& Sokoloff, D. D. (2010). phylogenetic placement of podolotus suggests independent origin of lomentaceous fruits in coronilla and hippocrepis (leguminosae: loteae) Pak. J. Bot., Special Issue (S.I. Ali Festschrift) 42, 11-25.

38. Endo, Y. \& Ohashi, H. (1997). Cladistic analysis of phylogenetic relationships among tribes cicereae, trifolieae, and vicieae (leguminosae). Amer. J. Bot, 84(4), 523-529. 
39. Käss, E. \& Wink, M. (1997). Phylogenetic relationships in the Papilionoideae (Family Leguminosae) based on nucleotide sequences of cpDNA ( $r b c \mathrm{~L})$ and ncDNA (ITS1 and 2). Molec. Phylogen. Evol, 8, 65-88.

40. Schulz, O. E. (1901). Monograhic der Gattung Melilotus. Bot. Jarb. Syst. 29, 660.

41. Survorov, V. V. (1950). Melilotus (Toun.) Adans Em. In flora of cultivated plants of the USSR, E. N. Sins Kaya, ed. 13(1), 426-627. Trans, from Russian as OTS, 60-51198.
42. Steele, K. P. \& Wojciechowski, M. F. (2003). Phylogenetic analyses of tribes Trifolieae and Vicieae, based on sequences of the plastid gene matK (Papilionoideae: Leguminosae). 355-370 in: Klitgaard, B.B. \& Bruneau, A. (eds.), Advances in legume systematics, part 10, Higher level systematics. Richmond, U.K.: Royal Botanic Gardens, Kew.

43. Gazara, M., Kamel, W. \& Haider, A. (2001). Cladistic analysis of the genera: Trifolium,Trigonella and Melilotus (Fabaceae: Papilionaceae) in Egypt. Egyp. J. Biol, 3, 161-170. 